\title{
Influence of Occupant Behavior for Building Energy Conservation: A Systematic Review Study of Diverse Modeling and Simulation Approach
}

\author{
Mohammad Nyme Uddin *(D), Hsi-Hsien Wei, Hung Lin Chi and Meng Ni *(D) \\ Department of Building and Real Estate, The Hong Kong Polytechnic University, Hung Hom, \\ Hong Kong 999077, China; hhwei@polyu.edu.hk (H.-H.W.); hung-lin.chi@polyu.edu.hk (H.L.C.) \\ * Correspondence: nymebd.uddin@connect.polyu.hk (M.N.U.); bsmengni@polyu.edu.hk (M.N.)
}

check for updates

Citation: Uddin, M.N.; Wei, H.-H.; Chi, H.L.; Ni, M. Influence of Occupant Behavior for Building Energy Conservation: A Systematic Review Study of Diverse Modeling and Simulation Approach. Buildings 2021, 11, 41. https://doi.org/ 10.3390/buildings11020041

Academic Editor: Brent Stephens Received: 23 November 2020

Accepted: 20 January 2021

Published: 26 January 2021

Publisher's Note: MDPI stays neutra with regard to jurisdictional claims in published maps and institutional affiliations.

Copyright: (c) 2021 by the authors. Licensee MDPI, Basel, Switzerland This article is an open access article distributed under the terms and conditions of the Creative Commons Attribution (CC BY) license (https:// creativecommons.org/licenses/by/ $4.0 /)$.
Abstract: Energy consumption in buildings depends on several physical factors, including its physical characteristics, various building services systems/appliances used, and the outdoor environment. However, the occupants' behavior that determines and regulates the building energy conservation also plays a critical role in the buildings' energy performance. Compared to physical factors, there are relatively fewer studies on occupants' behavior. This paper reports a systematic review analysis on occupant behavior and different modeling approaches using the Scopus and Science Direct databases. The comprehensive review study focuses on the current understanding of occupant behavior, existing behavior modeling approaches and their limitations, and key influential parameters on building energy conservation. Finally, the study identifies six significant research gaps for future development: occupant-centered space layout deployment; occupant behavior must be understood in the context of developing or low-income economies; there are higher numbers of quantitative occupant behavior studies than qualitative; the extensive use of survey or secondary data and the lack of real data used in model validation; behavior studies are required for diverse categories building; building information modeling (BIM) integration with existing occupant behavior modeling/simulation. These checklists of the gaps are beneficial for researchers to accomplish the future research in the built environment.

Keywords: building; occupant behavior; modeling; energy conservation

\section{Introduction}

Primary energy consumption has increased annually over the past decade. In particular, the building construction industry accounts for a substantial part of national and global energy consumption. In advanced nations or countries, buildings consume around 20-40\% of primary energy [1]. For example, buildings in the United Kingdom are accountable for $39 \%$ more energy consumption than in other European nations, which is slightly greater than the average energy consumption (37\%). Similarly, in the United States, the residential and commercial building sectors are responsible for approximately $41 \%$ of total energy usage, while $74 \%$ of the energy was used only for electricity in 2014 [2,3]. Hong Kong's buildings sectors contribute around $61 \%$ of total greenhouse gas emissions and also constitute about $90 \%$ of total energy consumption [2,4]. In Japan, the energy usage by buildings sectors accounted for $28 \%$ of total energy consumption; $13.2 \%$ and $14.8 \%$ in the commercial and residential industries, respectively. As stated by the International Energy Agency (IEA), The construction industry in Japan became the biggest energy end-user in 1999 [5,6]. Thus, energy consumption from construction and building operations is expected to boost by $1.5 \%$ per year over the $2012-2040$ period under the regular communal scenario, and it may double or even triple by 2050 as compared to 2010 [7-9].

According to the International Energy Agency-Energy in Buildings and Communities (IEA-EBC), Annex 53 [10], the overall building performance can be affected by six parameters, including building envelope, climate, energy and service systems, interior design 
conditions, building operation and maintenance, and occupant behavior [11,12]. Reviewing the current studies on building energy savings, one can find that most of these studies focus on so-called operational energy, including energy and service systems, and building and maintenance. However, moving beyond the technical approaches researched by these studies to buildings parse, changes in occupants' energy-use behavior have recently been found to be a relatively economical alternative in saving building energy. Occupant behavior (OB) is generally defined as the occupants' behavior towards building energy-related operations, i.e., controls of appliances such as lighting, HVAC (heating, ventilation, and air conditioning), or of windows, blinds, and so forth [13]. However, it has been observed that accurate predictions of $\mathrm{OB}$ have rarely been achieved as a result of their arising from the inherently different characteristics of individuals [14].

The building performance simulation (BPS) platform is an effective and cost-efficient option for analyzing and improving the building design and energy management, while a precise input of occupant behavior is fundamentally important $[15,16]$. Additionally, BPS can be used in the operating stage to check the real building performance and diagnose the building systems that may not work correctly. If refurbishment or re-modeling is required during the maintenance stage, the most energy-efficient retrofit model can be identified with BPS [17]. In other words, BPS can be used to assist the fault detection in the HVAC operation, and forecast the possible energy savings associated with system-level modifications and building redesign or retrofits, in order to increase the building's energy performance $[14,18]$. In contrast, a prevalent and significant source of error in existing BPS tools under realistic circumstances is the inaccurate or misleading input parameter associated with occupant behavior and building operation [18]. Another constraint is the inability of BPS to simulate realistic building performance [19]. In order to achieve accurate and reliable outcomes $[18,20]$, it is necessary to simulate the building under realistic circumstances, including occupant behavior (OB) [21]. In general, OB is mainly represented by the setting of indoor temperature, scheduling of equipment, lighting, and HVAC systems [22,23]. These are highly variable and totally unpredictable for individual occupants or groups of occupants $[24,25]$. These parameters also have a significant impact on real energy consumption, as well as overall building energy performance [11,23]. Nowadays, BPS incorporating occupant behavior has the ability to predict almost-real energy consumption [26]. Improving the knowledge of occupant behavior is therefore essential for assessing its influence on the overall output of the building [27]. In order to understand the energy-behavior interaction, the latest studies in residential and commercial buildings have found that occupants' behavior within an indoor atmosphere has a twofold impact on both the total building energy and the occupant's comfort with the quality of indoor environment [28]. Given to this energy-comfort connection, researchers no longer have the luxury of treating occupant-related factors as a boundary condition. In other words, the following of default assumptions about the behavior of occupants in energy modeling has disappeared. As such, in the latest simulation studies, the method of simulating occupant behavior has gained growing attention, with several approaches being applied to mimic the conduct of occupants in buildings energy research [29].

The building occupant is an important part in the built environment, and its eminence in building energy research has recently started to receive more attention [18]. Since occupant behavior has been revealed as one of the most critical parts for building energy conservation at the design and operation stage, several methodologies or approaches have been developed to identify and analyze the comprehensive behavior for allowing the utmost building energy conservation $[4,30,31]$. However, the influence of occupant behavior on building energy consumption is very complex, as it is reliant on several factors or determinants [32]. The building occupants provide the major, most cost-effective possibilities for energy efficiency and the largest co-benefits. Considering the importance of occupant behavior in building energy performance and the lack of a systematic review, this paper aims to provide a timely review of the state of the art literature on occupant behavior research. It is obviously difficult to capture a holistic understanding of occupant 
behavior and its influence on building energy conservation. Particularly, the following questions remain unanswered: (a) What is the current understanding of occupant behavior and influential determinants related to buildings' energy consumption? (b) What kind of drawbacks and limitations are involved in the existing occupant behavior modeling approach? (c) How has behavior research progressed and what are the further research gaps? This review study tries to address the above questions through a systematic review study described in the subsequent section.

Thus, the remaining parts of the study present the methodology adopted; the cooccurrence of keywords; an overview of the occupant behavior modeling approach with benefits and drawbacks; occupant behavior with regard to monitoring to obtain the relevant information; influential parameters; the challenges and opportunities of $\mathrm{OB}$ in building energy conservation.

\section{Methodology of Literature Review}

As shown in Figure 1, the methodology adopted in this study is built on a systematic review of the most relevant research that addresses the issues related to occupant behavior in terms of building energy conservation. This review includes relevant articles that are already published in peer-reviewed academic journals, while unpublished research works, conference papers, policy or industry reports, short communications, etc., are excluded. The proper justification behind this is that peer-reviewed articles are considered the most valuable sources of data or information, as greater academic precision is involved in their research publications [33]. The systematic search of the literature was conducted using the most popular search engine, namely the Scopus and Science Direct database, to retrieve the related articles for this review study. Moreover, Scopus has been preferred over other search engines, for example, Google Scholar and Web of Science, while to review the literature on a specific issue or topic Scopus has been considered as the best and most useful search engine [34].

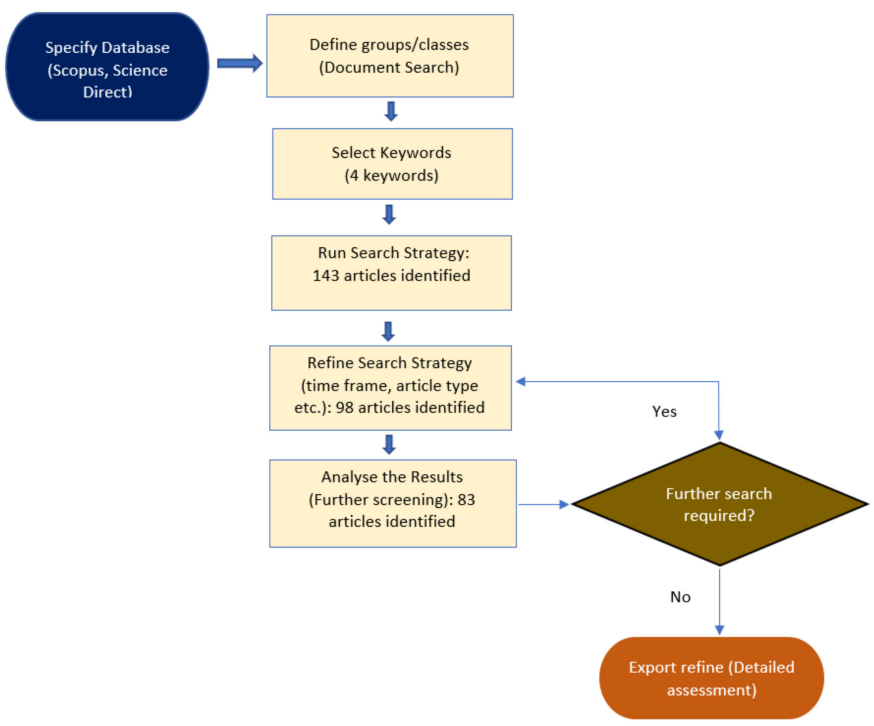

Figure 1. Flow chart of review study.

Using document type "Article or Review", date range "Published 2010 to 2019", and the "Article Title, Abstract, Keywords" section of Scopus, the search for articles relevant to occupant behavior modeling for building energy conservation was accomplished using the following keywords: "occupant behavior", "modeling", "building", "energy conservation". It is also noted that these keywords may not be very comprehensive, but they are helpful in finding a possible number of relevant articles for this analysis. The reason for selecting the period 2010-2019 is because the relevant research in the last decade was very active, especially in the last few years [11]. The initial search identified approximately 143 papers. 
With a focus on articles published in building-, energy-, and construction-related peerreviewed journals, 98 articles published in more than 28 different peer-reviewed journals were selected. Furthermore, several articles just mentioned the selected keywords in their title, abstract or keywords sections, and thus they are excluded. After the detailed screening, a total number of 83 articles (Scopus and Science Direct) were selected for further comprehensive analysis. The trend in different keyword combinations by year and journals from the Scopus database are shown in Figures 2-5.

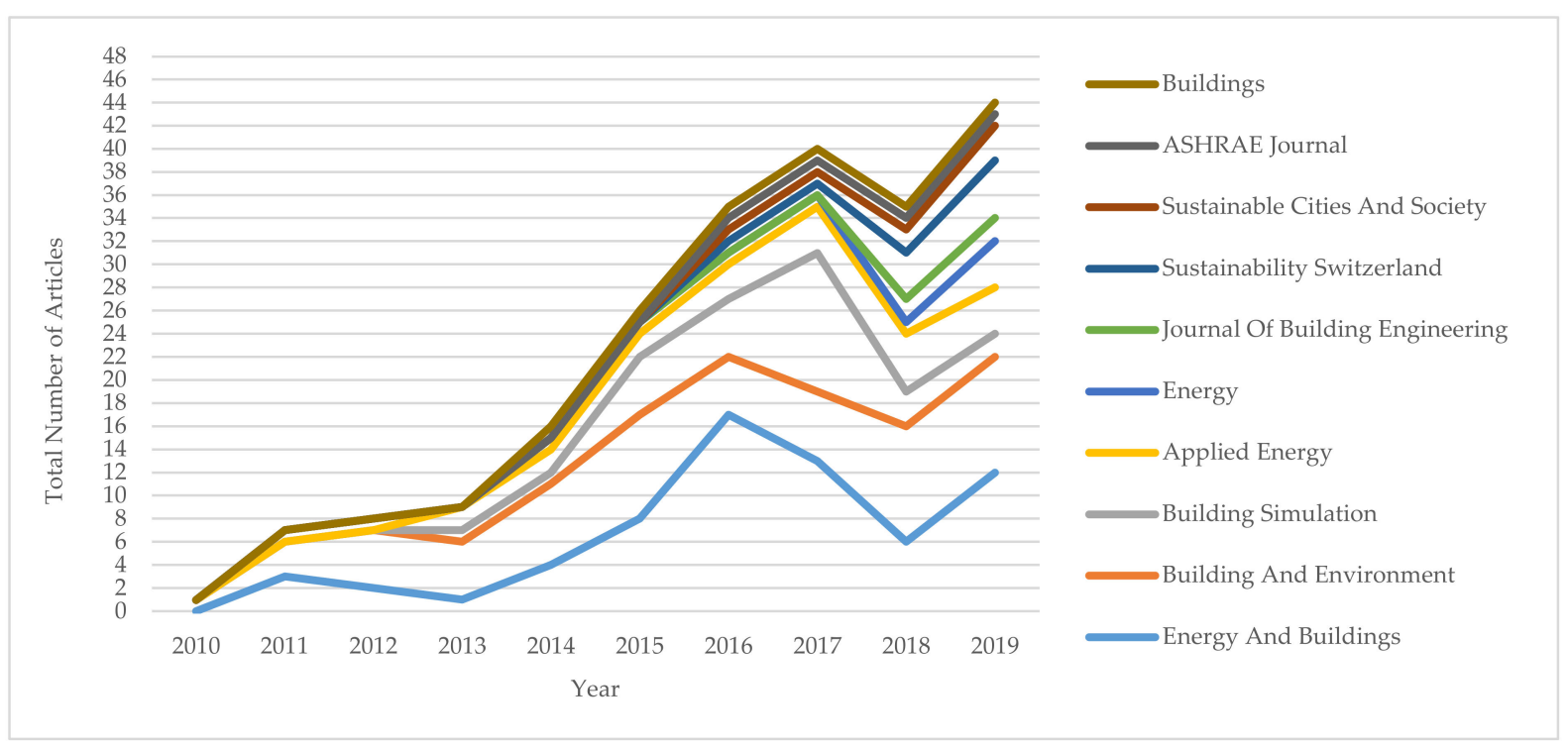

Figure 2. The trend in keyword "Occupant Behavior".

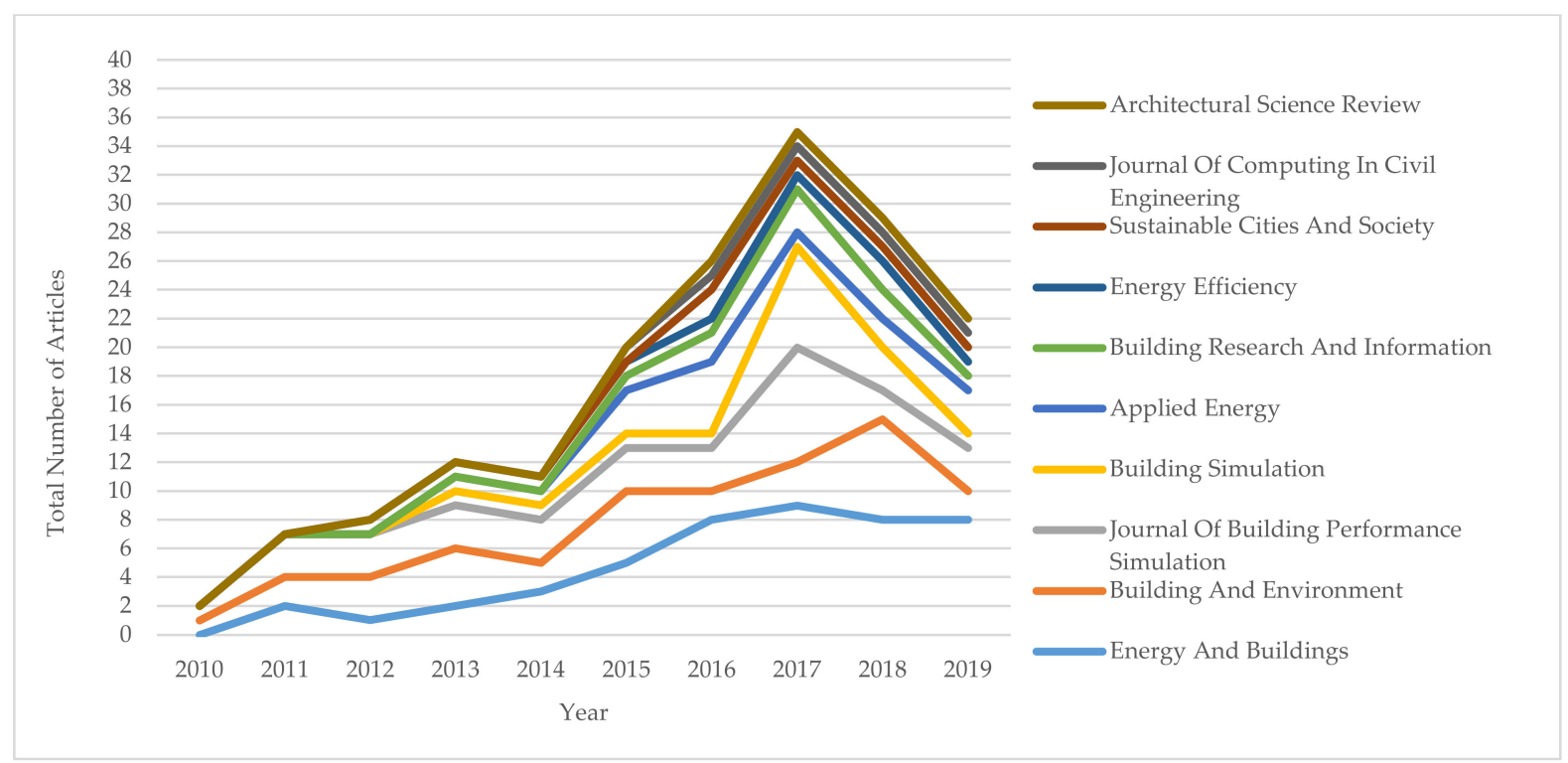

Figure 3. The trend in keywords "Occupant Behavior" and "Modeling". 


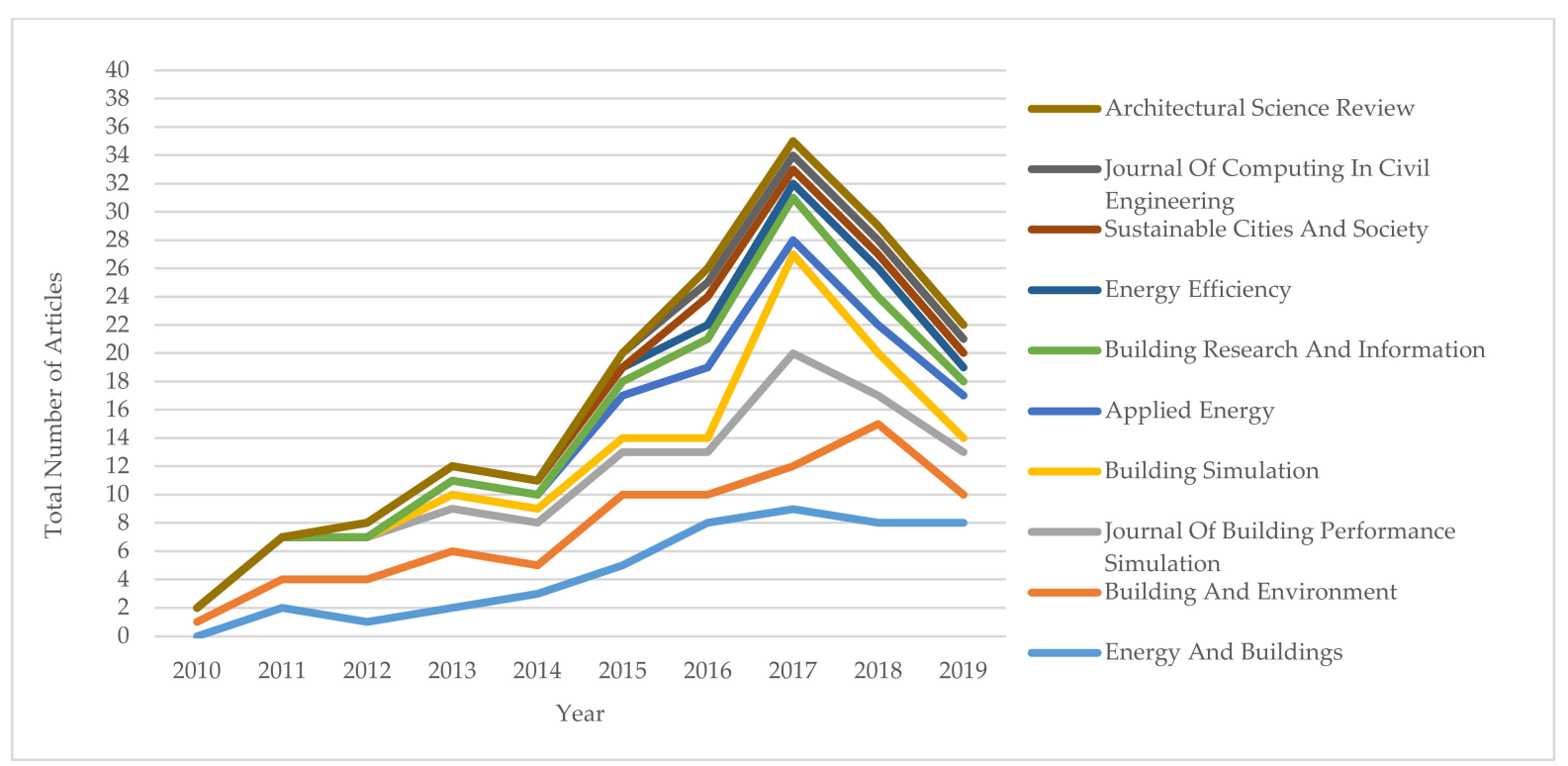

Figure 4. The trend in keywords "Occupant Behavior", "Modeling", and "Building".

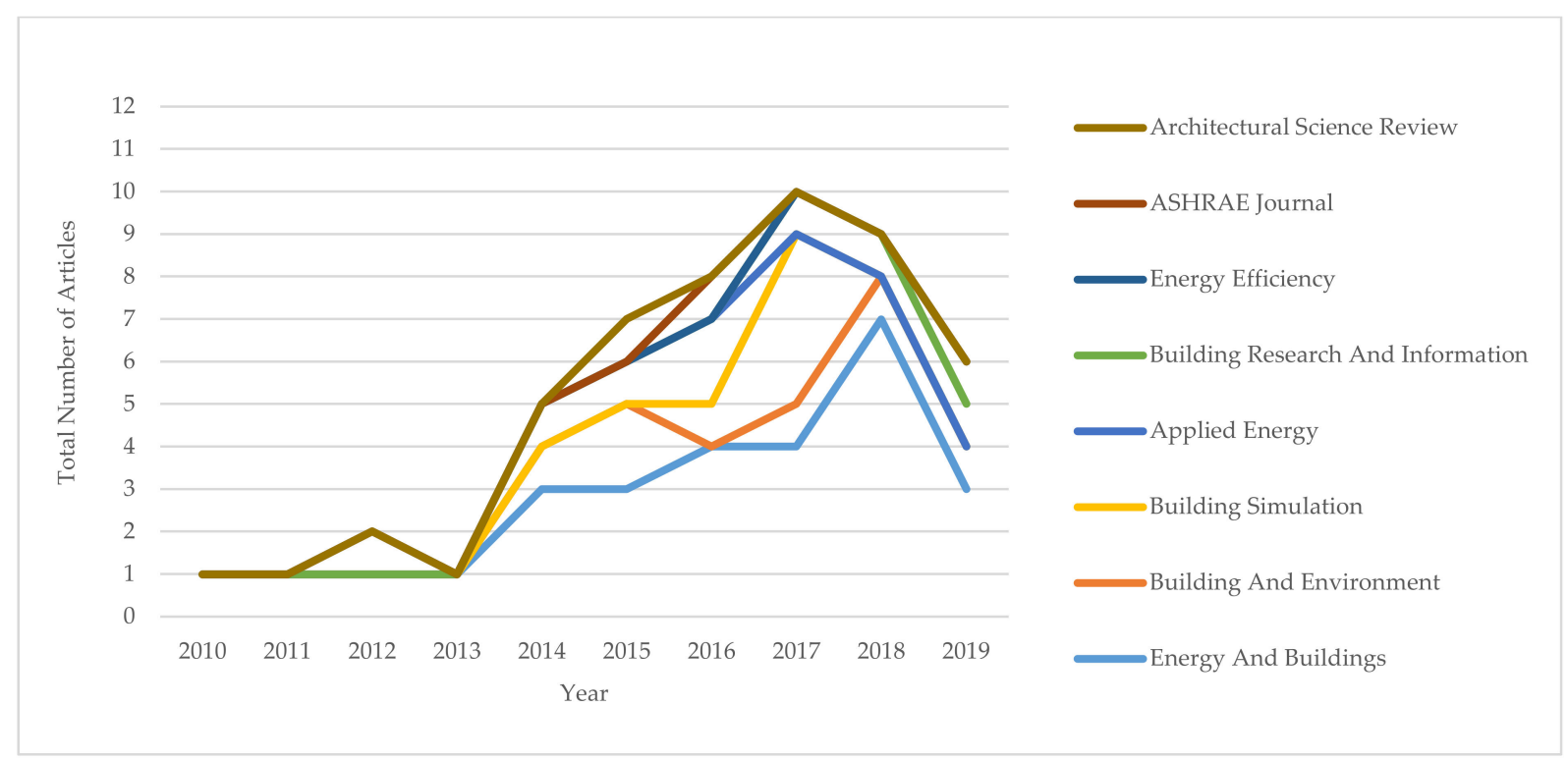

Figure 5. The trend in keywords "Occupant Behavior", "Modeling", "Building", and "Energy Conservation".

\section{Network of Countries/Regions and Co-Occurrence of Keywords}

A network was created showing the contribution and collaboration among various countries. The network diagram of countries was first generated using the VOSviewer software (version 1.6.15). The bigger the size of a node of a country, the higher the number of connections of the country to other countries in the network. The level of link among countries (shown as connecting lines), determined by the total link strength, depicts the collaboration strength among countries in publications. A thicker link between two countries indicates a stronger collaboration in terms of article publications (as shown in Figure 6). 


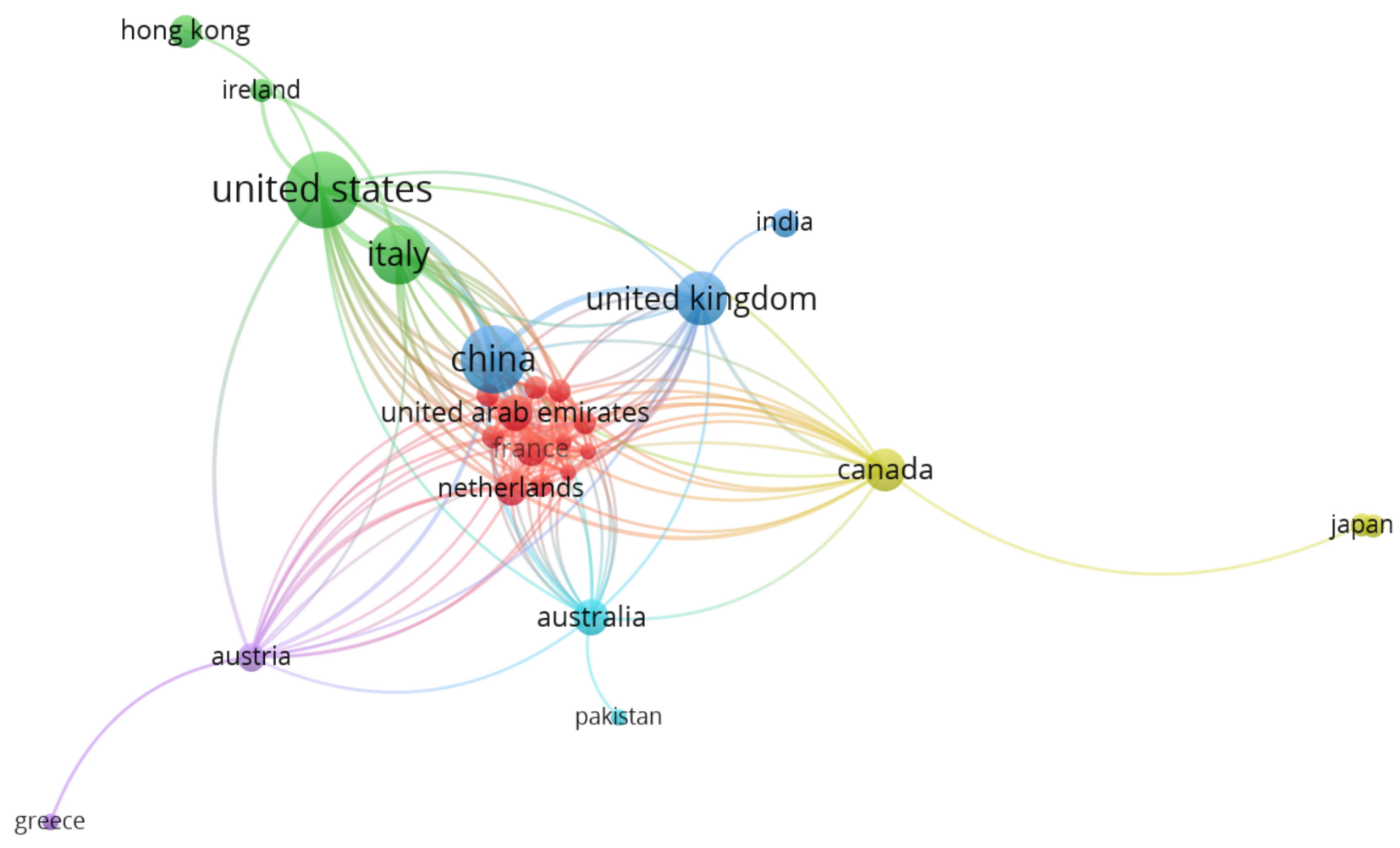

Figure 6. Network of countries/regions of research publication.

The network reveals interesting findings on research collaboration and the contribution of some countries. The United States of America (USA), UK, People's Republic of China, Italy, Canada, Austria, Australia, Hong Kong, UAE, Netherlands, Germany, and France, in descending order of degree values, are the top listed countries, with high degrees and high total link strength. These countries are the greatest contributors with a strong collaboration network with regard to the occupant behavior literature. The highest total link strengths between countries were observed among the following pairs: USA-People's R. China, USA-Italy, USA-UK, USA-UAE, USA-Canada, USA-Netherlands, USA-Hong Kong, People's R. China-Australia, People's R. China-Italy, People's R. China-Austria, People's R. China-France, Canada-Italy, Italy-Ireland, and UK-Italy. Except for People's $R$. China in these pairs, the other countries are developed countries. One possible reason for the strong link strength among these countries could be cross-country case studies and comparative studies.

Besides the countries/regions, this review study also performed a comprehensive keyword analysis using the VOSviewer tool. According to Zhao [35], keywords represent the main contents of an article and indicate the trend of the development of research topics. Similarly, Su and Lee [36] stated that a network and knowledge map of keywords depicts the knowledge structure of a particular field of research. It also reveals emerging elements and shows the dynamism of the knowledge structure. Prabhakaran et al. [37] mentioned that keywords show the "paradigm" and "paradigm shifts" in a field. Therefore, a keywords co-occurrence network was generated to determine the evolution of knowledge in occupant behavior studies during the last few decades. Figure 7 shows the network for only keywords that exceeded the occurrence frequency of 5 in the selected reviewed articles.

The size of the node is a depiction of the frequency of occurrence of the keywords, while the link and the total link strength attributes indicate, respectively, the number of links of an item with other items and the total strength of the co-occurrence links of a given keyword with other keywords. Totals of 48 nodes, 697 links, 1619 link strength and five clusters were generated. 


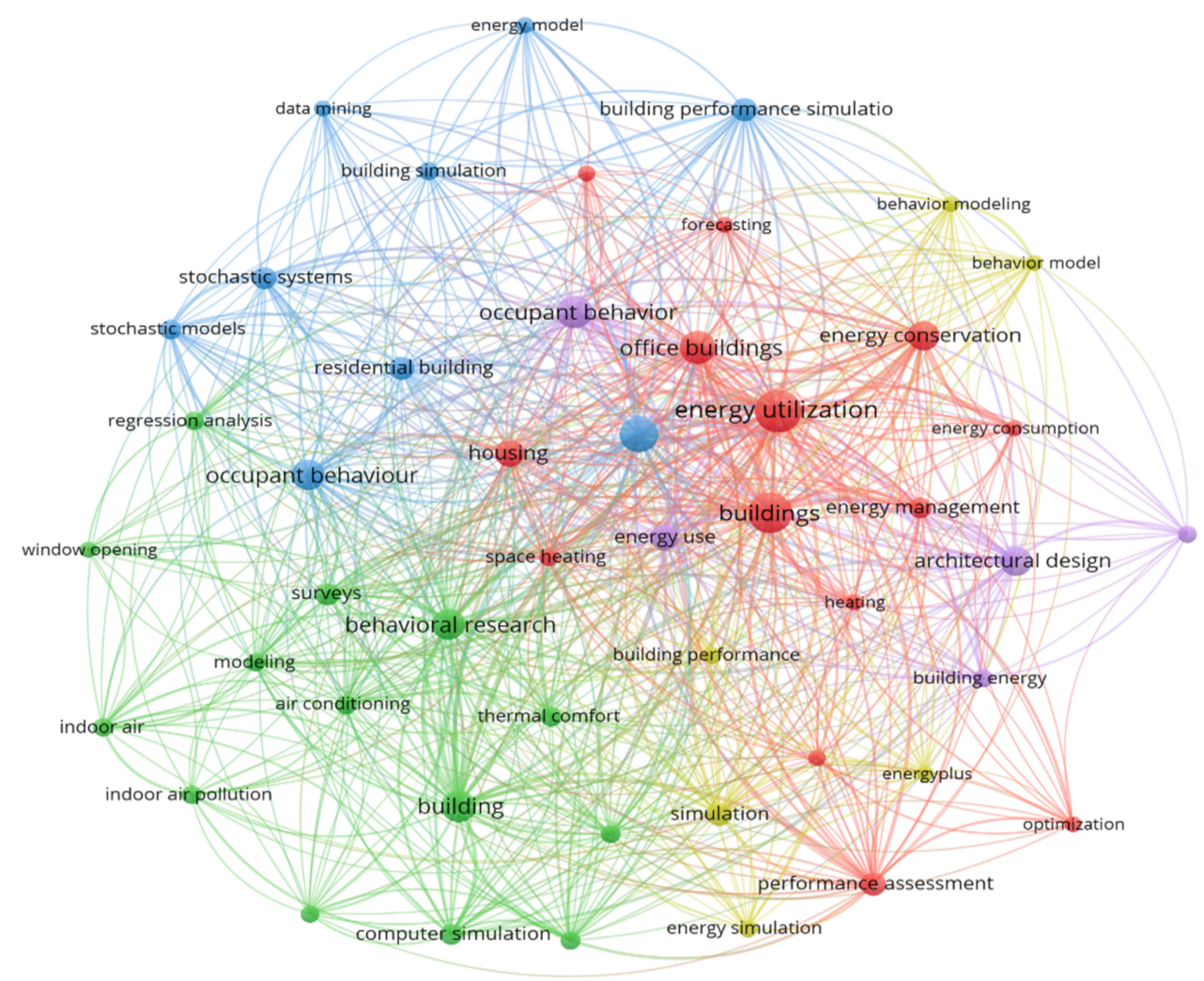

Figure 7. A network of co-occurrence of keywords from the selected articles.

During the last few decades, several keywords have garnered the attention of researchers and the industry, which are worth noting (as shown in Appendix A and the network in Figure 7). The 10 most frequent keywords include "Energy Utilization", "Buildings", "Energy Efficiency", "Occupant Behavior", "Office Building", "Behavioral Research", "Energy Conservation", "Architectural Design", "Performance Assessment", and "Simulation". The findings indicate that these keywords have received comparatively much attention in the occupant behavior literature. However, the other keywords had relatively low frequencies and total link strengths.

\section{Overview of Occupant Behavior (OB) Modeling and Simulation}

Building occupants insight not only hinders the completeness of the building energy model, as improper data of the occupants prompts errors in accurate building energy estimation. In spite of the fact that occupant behavior $(\mathrm{OB})$ is difficult to estimate because of the stochastic nature and randomness of people, it is important to investigate the common pattern of people's behavior and incorporate the data with the energy simulation model. With regards to the assembled condition and building energy conservation, generally in places of offices or residential buildings, the key driving variable that changes occupants ${ }^{\prime}$ behavior is their physical comfort as opposed to other paradigms, for example, social and economic concerns [34]. Furthermore, the climatic or environmental conditions where a resident/occupant lives will cause adaptive behavior, while proper energy use may be ignored [38]. Thus, a strong occupant behavior model can possibly reproduce sensible building users' responses to the diverse built environment. In the current modeling method, researchers typically pursue the specific methodology outlined in Figure 8. 


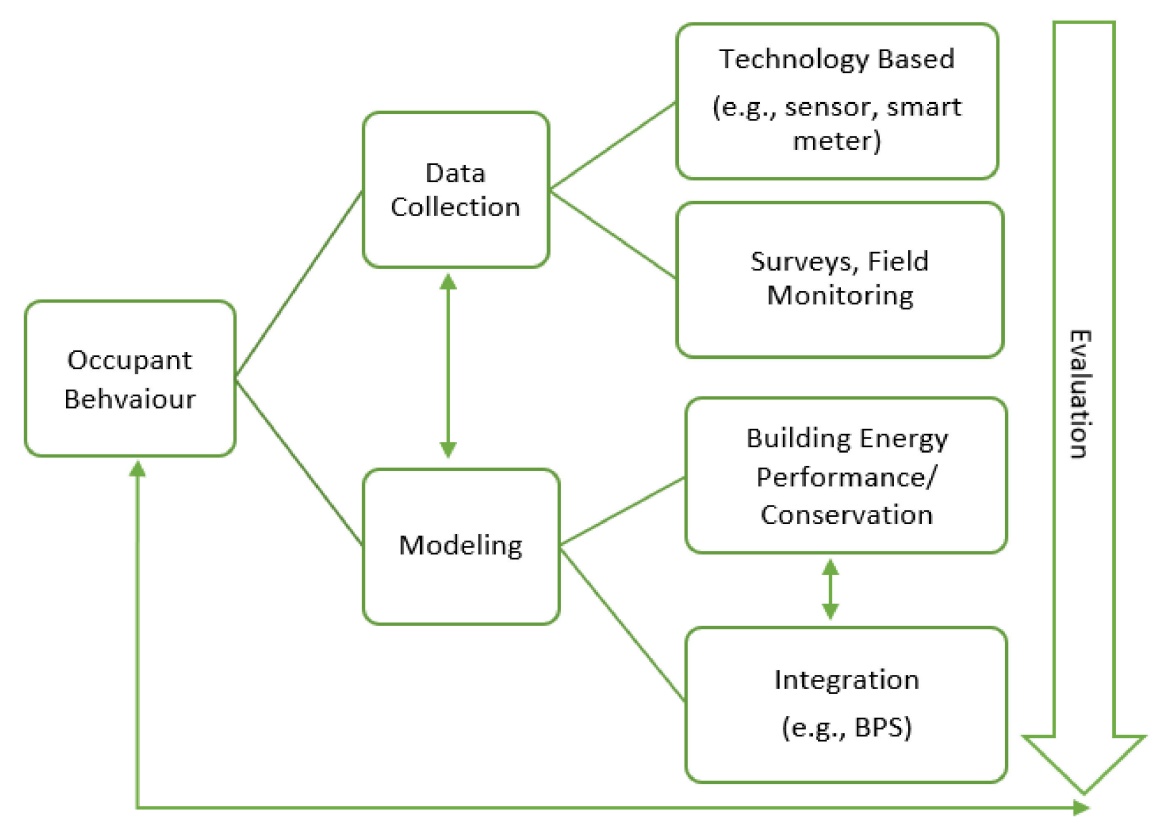

Figure 8. The structural framework of behavior modeling and simulation.

The primary step of occupant behavior research is the collection of behavior data and other relevant variables, together with behavior modeling. In this regard, information related to occupant behavior and environmental or climatic data is collected [39]. The data collection approaches may be performed using the technology-based (e.g., sensor, smart meter, etc.) or survey-based systems. In addition, the selection of data collection approaches should be associated with the purpose of specific behavioral models/approaches. Then, the collected data and the preferred behavioral model will be used to analyze the building energy performance/conservation along with the integration of existing BPS tools (e.g., EnergyPlus, DesignBuilder, etc.) towards further comprehensive analysis. Occasionally, quantitative investigation is applied in the signifying part to collect relationships between the outdoor and indoor ambient conditions, and additional actions and behavior, along with a lot of logical elements (i.e., modeling). Through these analyses, several outputs that correlate with occupant behavior and energy performance can be determined. In order to validate the developed models, several evaluation approaches to the models have been conducted. This includes several experimental sections that represent an intervention or sequence of simulation experiments for the model validation using real data. It usually involves a time interval data record and the observation of ambient environmental factors (i.e., temperature, $\mathrm{CO}_{2}$, etc.), occupant energy consumption, behavior patterns, etc.

However, it is important where the procedure has not been completed once through, yet is repeated. For example, throughout the model improvement or assessment, it might be revealed that inadequate data or information were gathered [22]. Similarly, a developed model may prove to be unrealistic, while it is implemented into a behavior simulation tool, as the model data sources are not promptly accessible. In order to confirm that the created models are reliable and powerful, a precise model assessment/evaluation needs to be performed [12]. To put it clearly, if issues are disclosed throughout a given phase, the researcher may need to rebuild and re-repeat the analysis.

\subsection{Classification of Occupant Behavior Modeling}

Occupant behavior (OB) can be addressed quantitatively at a specific range, regardless of its complex and stochastic nature, through a method for scientific models' development $[39,40]$. Due to the complexity of the occupant behaviors, researchers have tried to develop various behavior models in buildings through several approaches [41,42]. Papadopoulos and Azar [43] classified human behavior models into three different categories, such as the grey-box model built on the statistical and stochastic approach, the white-box 
model built on physical equations, and the black-box model, which is based on machine learning algorithms. According to Hong et al. [25], OB models can be classified into implicit models and explicit models. Implicit models deal directly with rules and regulations related to physical building systems (e.g., lights and windows), and include (i) probability calculations, (ii) statistical assessment, (iii) linear and logistical regression, (iv) occupancy-based control models (i.e., sub-hourly), and (v) Bayesian estimates. Explicit models address the rules and logics directly associated with the occupants, and include (i) Bernoulli process, (ii) agent-based modeling, (iii) Markov chains, and (iv) survival assessment. Moreover, from a detailed survey, one review [41] study offers model categorization in terms of whether the developed model is based on data, and therefore the authors categorized wide-ranging behavior models into data-driven and simulation-based approaches. In brief, modeling using data-driven methods involves an extensive amount of data to build a statistical model for selected occupant behaviors, although simulation-based energy models depend on empirical or pre-defined rules that control the occupant behavior configuration $[44,45]$. A comprehensive list of quantitative modeling for this review study is shown in Table 1.

Table 1. List of quantitative modeling for building occupant behavior (2010-2019).

\begin{tabular}{|c|c|c|c|c|c|c|c|c|}
\hline $\begin{array}{l}\text { Modeling } \\
\text { Approach }\end{array}$ & $\begin{array}{c}\text { Study Goal/ } \\
\text { Technology/Theme }\end{array}$ & $\begin{array}{l}\text { Building } \\
\text { Category }\end{array}$ & $\begin{array}{l}\text { Case/Study } \\
\text { Location }\end{array}$ & $\begin{array}{l}\text { Modeled/Targeted } \\
\text { Behaviors }\end{array}$ & Tools/Platform & $\begin{array}{l}\text { Occupants/ } \\
\text { Datasources }\end{array}$ & $\begin{array}{c}\text { Model } \\
\text { Validation? }\end{array}$ & References \\
\hline \multirow{7}{*}{$\begin{array}{l}\text { Probabilistic } \\
\text { or Stochastic } \\
\text { Modeling }\end{array}$} & $\begin{array}{l}\text { To identify seven } \\
\text { typical occupancy } \\
\text { patterns using } \\
\text { hierarchical } \\
\text { clustering. }\end{array}$ & Residential & Belgium & $\begin{array}{c}\text { Occupancy } \\
\text { sequences at }(1) \\
\text { home and } \\
\text { awake, (2) sleeping } \\
\text { or (3) absent. }\end{array}$ & $\begin{array}{c}\text { Not } \\
\text { Mentioned }\end{array}$ & $\begin{array}{c}\text { Belgian } \\
\text { Time-Use } \\
\text { Survey (TUS) } \\
\text { Household } \\
\text { Budget Survey } \\
\text { (HBS) }\end{array}$ & $\begin{array}{c}\text { Not } \\
\text { Mentioned }\end{array}$ & {$[46]$} \\
\hline & $\begin{array}{c}\text { Integrating } \\
\text { occupants' presence } \\
\text { and behavior data } \\
\text { with the urban energy } \\
\text { modeling tool. }\end{array}$ & Laboratory & Switzerland & $\begin{array}{c}\text { Occupants' } \\
\text { presence, opening } \\
\text { and closing } \\
\text { windows, raising } \\
\text { and lowering of } \\
\text { blinds }\end{array}$ & CitySim & Survey data & $\begin{array}{c}\text { Not } \\
\text { Mentioned }\end{array}$ & [47] \\
\hline & $\begin{array}{l}\text { Develop an approach } \\
\text { for suitable } \\
\text { recordings of } \\
\text { occupants' presence } \\
\text { and simulation of } \\
\text { single- to } \\
\text { multiple-persons } \\
\text { office environments. }\end{array}$ & Office & $\begin{array}{c}\text { San } \\
\text { Francisco }\end{array}$ & $\begin{array}{l}\text { Presence of } \\
\text { occupants }\end{array}$ & $\begin{array}{c}\text { Not } \\
\text { Mentioned }\end{array}$ & $\begin{array}{l}\text { Passive } \\
\text { infrared } \\
\text { sensors }\end{array}$ & $\begin{array}{c}\text { Not } \\
\text { Mentioned }\end{array}$ & [48] \\
\hline & $\begin{array}{l}\text { Modeled diary-based } \\
\text { individuals' daily } \\
\text { activities for } 24 \mathrm{~h}, \\
\text { starting and ending } \\
\text { at } 04: 00 \text { including } \\
\text { weekdays and } \\
\text { weekends. }\end{array}$ & Residential & Denmark & $\begin{array}{l}\text { Occupancy pattern, } \\
\text { energy-related } \\
\text { activities }\end{array}$ & $\begin{array}{c}\text { A } \\
\text { questionnaire, } \\
\text { a diary, and an } \\
\text { expenditure } \\
\text { booklet }\end{array}$ & $\begin{array}{c}\text { Danish } \\
\text { Time-Use } \\
\text { Survey (TUS) }\end{array}$ & $\begin{array}{c}\text { Not } \\
\text { Mentioned }\end{array}$ & [49] \\
\hline & $\begin{array}{l}\text { The application of } \\
\text { hidden Markov } \\
\text { models (HMMs) to } \\
\text { create methods for } \\
\text { indirect observations } \\
\text { of energy } \\
\text { consumption for } \\
14 \text { residences. } \\
\text { To estimate the }\end{array}$ & Residential & Spain & $\begin{array}{c}\text { Electricity } \\
\text { consump- } \\
\text { tion/Occupancy } \\
\text { pattern }\end{array}$ & Smart meter & $\begin{array}{l}\text { Occupant } \\
\text { survey }\end{array}$ & Yes & [50] \\
\hline & $\begin{array}{l}\text { predictive accuracy of } \\
\text { four sets of models } \\
\text { for window opening } \\
\text { behavior. }\end{array}$ & Residential & Denmark & Window opening & $\begin{array}{c}\text { Not } \\
\text { Mentioned }\end{array}$ & $\begin{array}{l}\text { Secondary } \\
\text { data }\end{array}$ & Yes & [51] \\
\hline & $\begin{array}{l}\text { Application of } \\
\text { probability } \\
\text { distribution for } \\
\text { occupancy dependent } \\
\text { input parameters } \\
\text { such as air change } \\
\text { rates, internal heat } \\
\text { gains. }\end{array}$ & Laboratory & Italy & HVAC Energy & $\begin{array}{c}\text { Not } \\
\text { Mentioned }\end{array}$ & Sensor & Calibration & [44] \\
\hline
\end{tabular}


Table 1. Cont.

\begin{tabular}{|c|c|c|c|c|c|c|c|c|}
\hline $\begin{array}{l}\text { Modeling } \\
\text { Approach }\end{array}$ & $\begin{array}{c}\text { Study Goal/ } \\
\text { Technology/Theme }\end{array}$ & $\begin{array}{l}\text { Building } \\
\text { Category }\end{array}$ & $\begin{array}{l}\text { Case/Study } \\
\text { Location }\end{array}$ & $\begin{array}{c}\text { Modeled/Targeted } \\
\text { Behaviors }\end{array}$ & Tools/Platform & $\begin{array}{c}\text { Occupants/ } \\
\text { Datasources }\end{array}$ & $\begin{array}{c}\text { Model } \\
\text { Validation? }\end{array}$ & References \\
\hline \multirow{7}{*}{$\begin{array}{l}\text { Statistical } \\
\text { Modeling }\end{array}$} & $\begin{array}{c}\text { To determine } \\
\text { behavioral patterns } \\
\text { associated with the } \\
\text { heating energy } \\
\text { consumption and } \\
\text { identify the } \\
\text { household and } \\
\text { building energy } \\
\text { characteristics. }\end{array}$ & Office & Netherlands & $\begin{array}{c}\text { Behavioral } \\
\text { Patterns, HVAC } \\
\text { systems }\end{array}$ & $\begin{array}{c}\text { Not } \\
\text { Mentioned }\end{array}$ & $\begin{array}{l}\text { A household } \\
\text { survey }\end{array}$ & $\begin{array}{c}\text { Not } \\
\text { Mentioned }\end{array}$ & [52] \\
\hline & $\begin{array}{l}\text { To construct a } \\
\text { multiple linear } \\
\text { regression model for } \\
\text { four specific } \\
\text { parameters. }\end{array}$ & Residential & Ireland & $\begin{array}{c}\text { Occupant } \\
\text { characteristics of } \\
\text { domestic electricity } \\
\text { consumption } \\
\text { patterns }\end{array}$ & Smart meter & Survey & $\begin{array}{c}\text { Not } \\
\text { Mentioned }\end{array}$ & [53] \\
\hline & $\begin{array}{l}\text { Models of occupants' } \\
\text { interactions with } \\
\text { windows and } \\
\text { window opening } \\
\text { behavior were judged } \\
\text { using a simulation } \\
\text { program. }\end{array}$ & Residential & Denmark & $\begin{array}{l}\text { Window opening } \\
\text { and closing }\end{array}$ & IDA ICE & $\begin{array}{l}\text { Secondary } \\
\text { data }\end{array}$ & $\begin{array}{c}\text { Not } \\
\text { Mentioned }\end{array}$ & [54] \\
\hline & $\begin{array}{c}\text { A new approach to } \\
\text { combine probabilistic } \\
\text { user profiles for both } \\
\text { thermostat set-points } \\
\text { and window opening } \\
\text { as well as } \\
\text { adjustments into a } \\
\text { building energy } \\
\text { model. }\end{array}$ & Residential & Denmark & $\begin{array}{l}\text { Thermostat and } \\
\text { window opening } \\
\text { occupant behavior }\end{array}$ & IDA ICE & $\begin{array}{l}\text { Field } \\
\text { monitoring } \\
\text { campaign, } \\
\text { sensor }\end{array}$ & $\begin{array}{c}\text { Not } \\
\text { Mentioned }\end{array}$ & [55] \\
\hline & $\begin{array}{l}\text { To predict the } \\
\text { occurrence and } \\
\text { frequency of } \\
\text { intermediate } \\
\text { activities during } \\
\text { office hours. }\end{array}$ & Office & Netherlands & $\begin{array}{c}\text { Intermediate } \\
\text { activity behavior in } \\
\text { an office }\end{array}$ & $\begin{array}{c}\text { Not } \\
\text { Mentioned }\end{array}$ & $\begin{array}{l}\text { Other } \\
\text { resources }\end{array}$ & $\begin{array}{c}\text { Not } \\
\text { Mentioned }\end{array}$ & [56] \\
\hline & $\begin{array}{l}\text { A model that gives } \\
\text { the probability of air } \\
\text { conditioning turn on, } \\
\text { turn off. }\end{array}$ & Residential & China & AC Operation & EnergyPlus & $\begin{array}{c}\text { Field } \\
\text { measurement, } \\
\text { temperature } \\
\text { sensor, } \\
\text { Reco APP }\end{array}$ & Yes & [57] \\
\hline & $\begin{array}{l}\text { To identify the } \\
\text { effectiveness and } \\
\text { potential of smart } \\
\text { meters and real-time } \\
\text { IHDs for reducing } \\
\text { household energy } \\
\text { consumption. }\end{array}$ & Residential & China & $\begin{array}{l}\text { Electricity } \\
\text { consumption } \\
\text { pattern in two } \\
\text { groups of } \\
\text { occupants }\end{array}$ & $\begin{array}{c}\text { Not } \\
\text { Mentioned }\end{array}$ & $\begin{array}{l}\text { IHD, smart } \\
\text { meter, and } \\
\text { on-site } \\
\text { installation }\end{array}$ & $\begin{array}{c}\text { Not } \\
\text { Mentioned }\end{array}$ & [58] \\
\hline \multirow{5}{*}{ Data Mining } & $\begin{array}{l}\text { A three-step data } \\
\text { mining framework to } \\
\text { discover occupancy } \\
\text { patterns in office } \\
\text { spaces. }\end{array}$ & Office & Germany & $\begin{array}{l}\text { Occupancy pattern } \\
\text { /schedule }\end{array}$ & RapidMiner & Sensor & $\begin{array}{c}\text { Not } \\
\text { Mentioned }\end{array}$ & [59] \\
\hline & $\begin{array}{l}\text { To investigate the } \\
\text { occupants behavior } \\
\text { for adjusting } \\
\text { thermostat settings } \\
\text { and heating system } \\
\text { for a housing } \\
\text { complex. }\end{array}$ & Residential & USA & $\begin{array}{l}\text { Occupant behavior } \\
\text { patterns (on/off } \\
\text { space heating) }\end{array}$ & $\begin{array}{l}\text { RapidMiner } \\
\text { Studio } 6.0\end{array}$ & Sensor/Manual & $\begin{array}{c}\text { Not } \\
\text { Mentioned }\end{array}$ & [40] \\
\hline & $\begin{array}{l}\text { A new methodology } \\
\text { for monitoring } \\
\text { energy consumption } \\
\text { and end-use loads to } \\
\text { build a review } \\
\text { system. }\end{array}$ & Residential & Japan & $\begin{array}{l}\text { Total energy } \\
\text { consumption }\end{array}$ & $\begin{array}{c}\text { Field } \\
\text { measurement, } \\
\text { a } \\
\text { questionnaire }\end{array}$ & $\begin{array}{c}\text { Secondary } \\
\text { data (Japan) }\end{array}$ & Yes & {$[60]$} \\
\hline & $\begin{array}{c}\text { To develop an } \\
\text { indirect data mining } \\
\text { approach using } \\
\text { occupant passive } \\
\text { behavior. }\end{array}$ & $\begin{array}{c}\text { Office } \\
\text { building }\end{array}$ & USA & $\begin{array}{c}\text { occupancy } \\
\text { schedules } \\
\text { HVAC operation }\end{array}$ & $\begin{array}{l}\text { Fitbit FlexTM } \\
\text { pedometer, } \\
\text { Bluetooth } \\
\text { Dongle }\end{array}$ & $\begin{array}{l}\text { Plugwise } \\
\text { wireless smart } \\
\text { meters }\end{array}$ & Yes & [61] \\
\hline & $\begin{array}{l}\text { To propose an } \\
\text { inexpensive and } \\
\text { minimally invasive } \\
\text { approach to } \\
\text { recognize the } \\
\text { behavioral data from } \\
\text { environmental } \\
\text { factors. }\end{array}$ & Residential & China & AC operations & $\begin{array}{l}\text { Algorithms } \\
\text { developed to } \\
\text { recognize the } \\
\text { AC operations }\end{array}$ & $\begin{array}{l}\text { Wireless data } \\
\text { collection } \\
\text { system (WiFi } \\
\text { gateway) }\end{array}$ & Yes & [62] \\
\hline
\end{tabular}


Table 1. Cont.

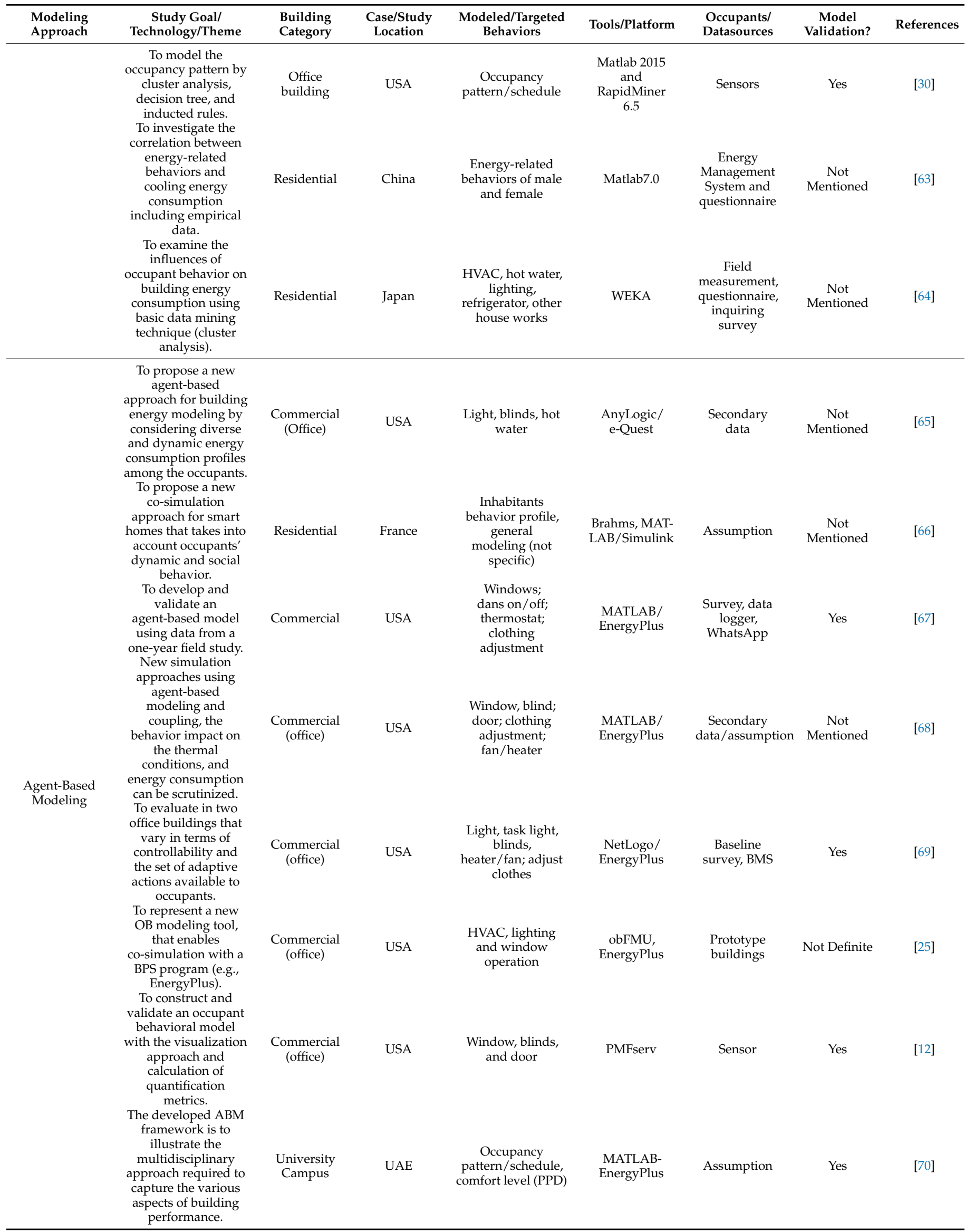


Table 1. Cont.

\begin{tabular}{|c|c|c|c|c|c|c|c|c|}
\hline $\begin{array}{l}\text { Modeling } \\
\text { Approach }\end{array}$ & $\begin{array}{c}\text { Study Goal/ } \\
\text { Technology/Theme }\end{array}$ & $\begin{array}{l}\text { Building } \\
\text { Category }\end{array}$ & $\begin{array}{l}\text { Case/Study } \\
\text { Location }\end{array}$ & $\begin{array}{c}\text { Modeled/Targeted } \\
\text { Behaviors }\end{array}$ & Tools/Platform & $\begin{array}{c}\text { Occupants/ } \\
\text { Datasources }\end{array}$ & $\begin{array}{c}\text { Model } \\
\text { Validation? }\end{array}$ & References \\
\hline & $\begin{array}{l}\text { To develop an } \\
\text { agent-based model as } \\
\text { regards students as } \\
\text { heterogeneous } \\
\text { occupants. }\end{array}$ & University & China & $\begin{array}{c}\text { Occupancy pattern } \\
\text { and appliance-use } \\
\text { behaviors }\end{array}$ & AnyLogic & $\begin{array}{c}\text { SIMS } \\
\text { intelligent } \\
\text { electricity } \\
\text { query system, } \\
\text { survey } \\
\text { questionnaire }\end{array}$ & Yes & {$[71]$} \\
\hline & $\begin{array}{l}\text { To propose a new } \\
\text { modeling framework } \\
\text { that incorporates BPS } \\
\text { in the ABM model by } \\
\text { using trained } \\
\text { regression surrogate } \\
\text { models. }\end{array}$ & Office & USA & $\begin{array}{c}\text { Energy use } \\
\text { attributes of } \\
\text { building occupants } \\
\text { and facility } \\
\text { managers, } \\
\text { uncertainty in } \\
\text { occupant actions }\end{array}$ & $\begin{array}{l}\text { MATLAB/ } \\
\text { EnergyPlus }\end{array}$ & $\begin{array}{l}\text { Prototype } \\
\text { buildings } \\
\text { developed by } \\
\text { US DOE }\end{array}$ & $\begin{array}{c}\text { Not } \\
\text { Mentioned }\end{array}$ & [42] \\
\hline & $\begin{array}{l}\text { A toolkit uses the } \\
\text { Building Controls } \\
\text { Virtual Test Bed } \\
\text { (BCVTB), an } \\
\text { agent-based model } \\
\text { with EnergyPlus. }\end{array}$ & Office & USA & HVAC, plug loads & $\begin{array}{c}\text { MATLAB, } \\
\text { BCVTB, } \\
\text { EnergyPlus }\end{array}$ & $\begin{array}{l}\text { Prototype } \\
\text { buildings } \\
\text { developed by } \\
\text { US DOE }\end{array}$ & $\begin{array}{c}\text { Not } \\
\text { Mentioned }\end{array}$ & {$[72]$} \\
\hline & $\begin{array}{l}\text { To evaluate the } \\
\text { impact of extreme } \\
\text { energy users on their } \\
\text { peers and energy } \\
\text { effectiveness of } \\
\text { commonly employed } \\
\text { interventions. }\end{array}$ & Office & USA & $\begin{array}{l}\text { Occupancy } \\
\text { interventions }\end{array}$ & Anylogic & $\begin{array}{l}\text { Survey, } \\
\text { CBECS }\end{array}$ & $\begin{array}{c}\text { Not } \\
\text { Mentioned }\end{array}$ & [73] \\
\hline & $\begin{array}{c}\text { To develop an } \\
\text { agent-based } \\
\text { computational model } \\
\text { for individual energy } \\
\text { consumption } \\
\text { patterns. }\end{array}$ & Residential & USA & $\begin{array}{l}\text { Peer networks in } \\
\text { buildings and } \\
\text { energy } \\
\text { conservation } \\
\text { behaviors of } \\
\text { occupants. }\end{array}$ & $\begin{array}{c}\text { Not } \\
\text { mentioned }\end{array}$ & $\begin{array}{l}\text { Secondary } \\
\text { data }\end{array}$ & Yes & [74] \\
\hline & $\begin{array}{l}\text { To recognize the gap } \\
\text { by suggesting a } \\
\text { multilayer ABM } \\
\text { approach that serves } \\
\text { as a test bed to } \\
\text { simulate and } \\
\text { optimize. }\end{array}$ & Commercial & USA & $\begin{array}{c}\text { Energy feedback } \\
\text { within social circles }\end{array}$ & Anylogic & $\begin{array}{l}\text { Secondary } \\
\text { data }\end{array}$ & Yes & [75] \\
\hline \multirow{5}{*}{$\begin{array}{l}\text { Others (BPS, } \\
\text { Data-Driven, } \\
\text { ANN, etc.) }\end{array}$} & $\begin{array}{c}\text { To perform a } \\
\text { numerical- } \\
\text { experimental } \\
\text { operation through } \\
\text { sophisticated } \\
\text { modeling. }\end{array}$ & Residential & Italy & $\begin{array}{l}\text { Human-based } \\
\text { energy retrofit } \\
\text { scenarios }\end{array}$ & EnergyPlus & $\begin{array}{c}\text { Field } \\
\text { monitoring } \\
\text { and occupants' } \\
\text { survey }\end{array}$ & $\begin{array}{l}\text { Calibrated } \\
\text { validated }\end{array}$ & [76] \\
\hline & $\begin{array}{l}\text { To propose an online } \\
\text { learning-based } \\
\text { control strategy along } \\
\text { with its design } \\
\text { method including } \\
\text { four domains (e.g., } \\
\text { time, indoor and } \\
\text { outdoor climates, and } \\
\text { occupant behavior). }\end{array}$ & Office & Singapore & HVAC systems & $\begin{array}{l}\text { Advanced } \\
\text { algorithms }\end{array}$ & Sensors & Yes & [77] \\
\hline & $\begin{array}{l}\text { It recognizes the } \\
\text { energy consequences } \\
\text { of conventional } \\
\text { approaches to } \\
\text { occupant's behavior } \\
\text { modeling. }\end{array}$ & Office & Canada & $\begin{array}{l}\text { People, lighting, } \\
\text { and equipment } \\
\text { profiles }\end{array}$ & $\begin{array}{l}\text { SketchUp, } \\
\text { OpenStudio, } \\
\text { MATLAB } \\
\text { R2017a }\end{array}$ & Questionnaire & $\begin{array}{c}\text { Not } \\
\text { Mentioned }\end{array}$ & [78] \\
\hline & $\begin{array}{l}\text { To recommend an } \\
\text { integrative modeling } \\
\text { approach for energy } \\
\text { consumption } \\
\text { behaviors in the } \\
\text { residential } \\
\text { background. }\end{array}$ & Residential & Portuguese & $\begin{array}{l}\text { Total energy } \\
\text { consumption } \\
\text { behavior }\end{array}$ & $\begin{array}{l}\text { Energy plus/ } \\
\text { DesignBuilder }\end{array}$ & $\begin{array}{l}\text { Time-of-use } \\
\text { survey of } \\
\text { Portuguese } \\
\text { households }\end{array}$ & Yes & [79] \\
\hline & $\begin{array}{l}\text { To develop a } \\
\text { framework for } \\
\text { extracting relevant } \\
\text { data about the } \\
\text { uncertainties relating } \\
\text { to occupant profiles } \\
\text { of heating energy } \\
\text { consumption. }\end{array}$ & Residential & Canada & Space heating & $\begin{array}{l}\text { MATLAB } \\
\text { Simulink }\end{array}$ & Sensor & $\begin{array}{c}\text { Not } \\
\text { Mentioned }\end{array}$ & [80] \\
\hline
\end{tabular}


Table 1. Cont.

\begin{tabular}{|c|c|c|c|c|c|c|c|c|}
\hline $\begin{array}{l}\text { Modeling } \\
\text { Approach }\end{array}$ & $\begin{array}{c}\text { Study Goal/ } \\
\text { Technology/Theme }\end{array}$ & $\begin{array}{l}\text { Building } \\
\text { Category }\end{array}$ & $\begin{array}{l}\text { Case/Study } \\
\text { Location }\end{array}$ & $\begin{array}{l}\text { Modeled/Targeted } \\
\text { Behaviors }\end{array}$ & Tools/Platform & $\begin{array}{l}\text { Occupants/ } \\
\text { Datasources }\end{array}$ & $\begin{array}{c}\text { Model } \\
\text { Validation? }\end{array}$ & References \\
\hline & $\begin{array}{c}\text { To construct a } \\
\text { building occupant } \\
\text { behavior model using } \\
\text { simulation } \\
\text { approaches as well as } \\
\text { estimating the } \\
\text { potential energy } \\
\text { savings. }\end{array}$ & Office & USA & $\begin{array}{l}\text { Lighting energy } \\
\text { consumption }\end{array}$ & DeST software & Data portal & Calibration & [81] \\
\hline & $\begin{array}{l}\text { To assess the energy } \\
\text { performance and } \\
\text { comfort indices of the } \\
\text { building and } \\
\text { recognize the reasons } \\
\text { for malfunction. }\end{array}$ & Residential & Hungary & $\begin{array}{c}\text { Energy } \\
\text { performance and } \\
\text { comfort indices }\end{array}$ & IDA ICE & $\begin{array}{l}\text { Self-reported } \\
\text { surveys, } \\
\text { occupancy } \\
\text { sensors, and } \\
\text { fan-coil }\end{array}$ & Calibration & [82] \\
\hline & $\begin{array}{l}\text { A centralized system } \\
\text { to consider } \\
\text { energy-efficient } \\
\text { profiles by } \\
\text { considering solar } \\
\text { energy and high-level } \\
\text { services for hot water } \\
\text { systems. }\end{array}$ & Residential & China & $\begin{array}{l}\text { Domestic hot water } \\
\text { (DHW) system }\end{array}$ & $\begin{array}{c}\text { Not } \\
\text { mentioned }\end{array}$ & Survey & Yes & [83] \\
\hline & $\begin{array}{l}\text { To develop an } \\
\text { activity-based (e.g., } \\
\text { socio-demographic } \\
\text { and economic } \\
\text { attributes) framework } \\
\text { for quantifying } \\
\text { occupant energy } \\
\text { consumption } \\
\text { behavior. }\end{array}$ & Residential & France & $\begin{array}{l}\text { Domestic energy } \\
\text { consumption }\end{array}$ & $\begin{array}{c}\text { Not } \\
\text { mentioned }\end{array}$ & $\begin{array}{c}\text { National } \\
\text { statistical data }\end{array}$ & Yes & [84] \\
\hline & $\begin{array}{l}\text { To establish an } \\
\text { engineering-based } \\
\text { bottom-up model for } \\
\text { cooling energy } \\
\text { consumption. }\end{array}$ & Residential & China & $\begin{array}{l}\text { Cooling energy } \\
\text { consumption }\end{array}$ & DeST & $\begin{array}{l}\text { Survey, case } \\
\text { monitoring }\end{array}$ & $\begin{array}{c}\text { Not } \\
\text { Mentioned }\end{array}$ & [85] \\
\hline & $\begin{array}{l}\text { To improve the } \\
\text { accuracy in the } \\
\text { energy simulation } \\
\text { process by } \\
\text { considering the } \\
\text { occupancy data to } \\
\text { calibrate the energy } \\
\text { model. }\end{array}$ & Residential & Hong Kong & $\begin{array}{c}\text { Occupant schedule, } \\
\text { devices, } \\
\text { air-conditioners, } \\
\text { windows, lights, } \\
\text { domestic hot water, } \\
\text { and cooking }\end{array}$ & $\begin{array}{l}\text { DesignBuilder } \\
\text { and } \\
\text { EnergyPlus }\end{array}$ & $\begin{array}{c}\text { Questionnaire } \\
\text { survey }\end{array}$ & Yes & [86] \\
\hline & $\begin{array}{l}\text { To evaluate the } \\
\text { building energy } \\
\text { performance and } \\
\text { construct a reliable } \\
\text { simulation model for } \\
\text { energy- and } \\
\text { cost-efficient retrofit } \\
\text { design. }\end{array}$ & Residential & UK & $\begin{array}{c}\text { Occupancy profile, } \\
\text { energy } \\
\text { consumption } \\
\text { patterns, } \\
\text { thermal comfort }\end{array}$ & DesignBuilder & $\begin{array}{c}\text { A } \\
\text { questionnaire, } \\
\text { structured } \\
\text { interviews, } \\
\text { data loggers }\end{array}$ & $\begin{array}{c}\text { Not } \\
\text { Mentioned }\end{array}$ & [87] \\
\hline & $\begin{array}{l}\text { To investigate the role } \\
\text { of occupant } \\
\text { behavior for } \\
\text { supporting } \\
\text { decision-makers } \\
\text { dealing with the } \\
\text { renovation strategies. } \\
\text { Introduce a }\end{array}$ & Residential & Italy & $\begin{array}{c}\text { Thermostat, } \\
\text { heating system, } \\
\text { building } \\
\text { characteristics }\end{array}$ & DeST & $\begin{array}{l}\text { Surveys and } \\
\text { interviews, } \\
\text { observations, } \\
\text { reading from } \\
\text { meters and } \\
\text { statistics }\end{array}$ & Yes & [38] \\
\hline & $\begin{array}{l}\text { simulation approach } \\
\text { to estimate five } \\
\text { typical occupant } \\
\text { behavioral actions for } \\
\text { potential energy } \\
\text { savings. }\end{array}$ & Office & USA & $\begin{array}{c}\text { Occupancy } \\
\text { schedule, lighting, } \\
\text { plug load, HVAC } \\
\text { control, window } \\
\text { control }\end{array}$ & $\begin{array}{l}\text { EnergyPlus, } \\
\text { Occupancy } \\
\text { Simulator }\end{array}$ & Site survey & $\begin{array}{c}\text { Not } \\
\text { Mentioned }\end{array}$ & [22] \\
\hline & $\begin{array}{l}\text { To examine the } \\
\text { impact of physical } \\
\text { and behavioral } \\
\text { variables for energy } \\
\text { saving from the } \\
\text { retrofitting protected } \\
\text { housings. }\end{array}$ & Residential & London & $\begin{array}{l}\text { Energy-saving } \\
\text { from selected } \\
\text { housing retrofit }\end{array}$ & IESVE & $\begin{array}{l}\text { Existing } \\
\text { models and } \\
\text { the literature }\end{array}$ & Calibration & [88] \\
\hline & $\begin{array}{l}\text { To explore the } \\
\text { occupant factors that } \\
\text { influence the energy } \\
\text { consumption of a } \\
\text { case building in } \\
\text { Seoul, Tokyo, and } \\
\text { Hong Kong under the } \\
\text { climatic changes. }\end{array}$ & Office & $\begin{array}{l}\text { Hong Kong, } \\
\text { Japan, and } \\
\text { South Korea }\end{array}$ & HVAC energy & $\begin{array}{l}\text { EnergyPlus } \\
\text { Runtime } \\
\text { Language (Erl) }\end{array}$ & $\begin{array}{l}\text { Prototype } \\
\text { building } \\
\text { model } \\
\text { developed by } \\
\text { US DOE }\end{array}$ & $\begin{array}{c}\text { Not } \\
\text { Mentioned }\end{array}$ & [89] \\
\hline
\end{tabular}




\subsection{Brief Review of Existing Quantitative Modeling Approach}

\subsubsection{Probabilistic or Stochastic Modeling}

Probabilistic/Stochastic models capture and represent the probability that particular behavior occurs reliant on recorded or statistical information [46]. In general, there are three kinds of probabilistic or stochastic occupant's behavior models: Bernoulli process [47], Markov chain [48,90], and survival analysis [49]. All three models have been used to address both occupant movement and action to control their building interior condition. The Bernoulli process is a sequence of Bernoulli random variables, and its occupancy model predicts the probability of finding a space occupied as a function of the time of day. Usually, Markov chain (MC) is mostly used as a probabilistic model [91]. It is a time series procedure wherein all conditions of the framework can be straightforwardly observed. Here, the future condition or state depends on the current state, and is not influenced by past condition or state. Another model is called the hidden Markov model (HMM), which accepts the potential conditions of a framework connected in a general Markov chain (MC). However, the conditions of the framework are hidden from direct perception; rather, every framework state is related to a probability distribution with a lot of noticeable factors. Several researchers have employed MC models to represent the occupant's status and personal behavior standards. For instance, Liisberg et al. [50] used the hidden Markov model (HMM) to represent the occupant behavior that relied on indirect perceptions. Their study of typical probability reports as a function of time duration per day recognized four different occupant behavior profiles. Additionally, survival analysis (SA) is commonly used to evaluate the time-period of a state or events prior to a change, and it can be utilized to assess to what extent a building is probably going to be unaffected by building inhabitants or occupants [92]. Because of the randomness and occupant behavior disparity, stochastic or probabilistic models are more appropriate, as far as applicability and validity is concerned, for explaining the open coordination among the residents and building contexts than a deterministic or fixed modeling approach [51]. According to Gunay et al. [93], existing building performance simulations (BPSs) offer an integration opportunity with probabilistic or stochastic governing models. As such, this technique could be used to test the effect of occupant energy behavior. However, the problems of this technique cannot be neglected. This modeling approach is best suited for occupant longterm schedule formation or prediction. Comprehensive behaviors or occupant statistics are not explored with this technique [41].

\subsubsection{Statistical Modeling}

Statistical modeling is commonly built by constructing the numerical connection between occupant's behavior and indoor/outdoor conditions, energy utilization or time duration. Its outcomes are interconnected by the occupancy state or the probability of observed behavior for a particular time [94]. This modeling can be directed to recognize the patterns of behavior in buildings [52,53]. Fabi and other researchers [54,95] incorporated the approaches to understanding the two-type behaviors in a commercial building (e.g., office), such as light turning on/off and window-opening behaviors [55]. Moreover, a common statistical model was used in a previous research study [95] where logistic regression has been used to examine the impact of human thermal motives on various types of behavior, such as doors, windows, and blinds on/off status. The researchers found that indoor conditions, such as temperature $\left({ }^{\circ} \mathrm{C}\right)$, and $\mathrm{CO}_{2}(\mathrm{ppm})$, are more superior indicators than the outdoor conditions as driving factors of occupant energy behavior. Statistical modeling is a very common and conventional technique in occupant behavior modeling. In general, this practice is frequently used to analyze the relationship or connectivity between the building occupant behavior and numerous dynamic environmental conditions, i.e., indoor temperature $\left({ }^{\circ} \mathrm{C}\right), \mathrm{CO}_{2}$ (ppm) and relative humidity (\%). However, statistical analysis needs to be upgraded from different perspectives. Firstly, this system is only confined to one or two fixed categories of behavior analysis; for example, the status of a light switch on/off and window opening [51]. Even though this technique is straightforward and worthwhile, it is 
problematic to develop a comprehensive/wide-ranging model, as is further incorporation with the building energy simulation tools (e.g., EnergyPlus). Secondly, it does not matter in what way the higher probability is forecasted for the occupant behavior pattern, but in the real-life system, the occupant may behave in or follow another pattern, depending on individual mindset and general circumstances [2,56,92]. As such, incorporating truebehavior data (i.e., real data) into the statistical methods will be a better strategy for identifying the human behaviors in buildings.

\subsubsection{Data Mining Technique}

The data mining approach has been utilized in several of the latest investigations on human behavior $[40,59]$. It is the process of discovering patterns in a large data set. Usually, it requires an enormous database and immense information storage when used in behavior investigation [60]. The usage of data mining to describing human behavior trends, and its application in the study of building energy performance, are increasing. D'Oca and Hong [59] employed a three-phase data mining process that involves occupant status data sets from 16 offices in Frankfurt, Germany. It offered some insights into understanding an occupancy profile for the office occupants. The main advantage of this technique is data collection and management, which is easy to execute. From the earlier investigation, only occupancy or energy consumption $(\mathrm{kWh})$ data were recorded. The enormous amounts of data on building energy consumption and the energy used by individual appliance have become accessible. Zhao et al. [61] built up an "indirect" real-world data mining technique employing office appliances' energy consumption as a representative (i.e., proxy data) for occupants" "passive" behavior. Their study found that the average level of specifically categorized individual behavior occurrences was 90.29\%. Additionally, their experimental outcome indicated a genuinely stable occupancy pattern, while taking into account a wide variety of individual behaviors of using a piece of office equipment or an appliance. Whereas a great level of precision in standard behavior profiling or prediction can be accomplished, the implementation of this technique is limited to occupancy and appliances usage in individual buildings, potentially because of inadequate information and limited access to other behavioral and energy consumption data [62]. In most investigations to date, just domestic energy consumption data have been utilized for data mining studies of households' standard behavior patterns [16]. This method is intended to overcome the weaknesses of the previously mentioned techniques, especially when managing enormous data streams, by suggesting reliable occupant behavior models with the great potential for a quick examination and better replication $[30,59,96]$. In the opposite sense to the data mining approaches, agent-based modeling (ABM), a simulation-based approach that is usually built on real buildings, has been initiated within the occupant-centered virtual environment. As a powerful simulation-based system, recently ABM has become most popular for the occupant behavior modeling approach in the built environment.

\subsubsection{Agent-Based Modeling (ABM)}

In the past, researchers have applied much effort in modeling buildings' occupant behavior using various methodologies. One of the methodologies is the application of the agent-based modeling concept, which could be appropriated for behavioral prediction from the occupant's individual level to a group level [16]. ABM is a simulation-based framework that consists of single or multiple autonomous actors, called "agents", which interact with each other and their exterior/interior environmental state according to definite behavior rules. Labeodan et al. [97] also described ABM application in multi-agent structures, which included self-ruling agents, to simulate agents' interactions or relationships with one another within the environments under definite rules and directions. Such rules are essential to the energy simulation process, as they specifically characterize how and when agents interact or collaborate with each other by following the conditions within the environments. ABM has been implemented for building occupant interaction by Lee and Malkawi [68]. The study simulated different occupant behaviors in an office 
building. They analyzed five explicit behaviors: adjusting clothes level, activity level, space heater/individual fan use, window use and blind use. The main purpose of this study was to evaluate how an agent balances the dynamic thermal variations in a prototype office space to improve both energy savings and comfort. This methodology permits the incorporation of ABM models in both behavior and building energy execution, and it might be utilized as an integrated simulation approach for occupant energy behavior in commercial buildings [70]. Additionally, in a behavioral study, ABM has the ability to manage the uncertainties of the real situation [12]. Likewise, all parts of an agent in $\mathrm{ABM}$ could be represented with the goal that the agents act and think like a human. Nevertheless, inadequacies or limitations remain as the application of the ABM model to building occupant behavior studies is still at the promotion stage. In the future, the completeness and comprehensiveness of ABM-based models must be technologically up-to-date [41]. Furthermore, most of the previous studies that implemented the ABM approach remained on simulation data only, with no or proper validation [12,39]. As one of the crucial purposes of promoting occupant behavior is to reduce the difference between simulated and real energy consumption, this discrepancy cannot be neglected.

\subsection{Comparison of the Different Modeling Approaches}

Since occupant behavior has been revealed as one of the most critical factors for building energy conservation at the design and operation stages, several methodologies or approaches have been presented to identify and analyze the maximum building energy conservation. Prominently, it might not be resolved that one specific technique/approach is more suitable than the other approach. However, it is essential to understand the benefits and drawbacks of all approaches, as well as the flexibility of the system. A brief comparative review of the above-mentioned four approaches for the occupant behavior study has been listed in Table 2 .

Table 2. A brief comparison of the different modeling approaches.

\begin{tabular}{|c|c|c|c|c|c|}
\hline $\begin{array}{l}\text { Modeling } \\
\text { Approach }\end{array}$ & $\begin{array}{c}\text { Most Suitable Building } \\
\text { Type (s) }\end{array}$ & $\begin{array}{l}\text { Key Application/ } \\
\text { Modeling Purpose }\end{array}$ & $\begin{array}{l}\text { Real-Time Modeling } \\
\text { Capability }\end{array}$ & $\begin{array}{c}\text { Incorporation with } \\
\text { Simulation (i.e., with } \\
\text { BPS) }\end{array}$ & $\begin{array}{l}\text { Additional } \\
\text { Remarks }\end{array}$ \\
\hline $\begin{array}{l}\text { Probabilistic or } \\
\text { stochastic model }\end{array}$ & Commercial & $\begin{array}{c}\text { For better capturing } \\
\text { and representing of } \\
\text { the probability that a } \\
\text { specific behavior } \\
\text { occurs dependent on } \\
\text { recorded or statistical } \\
\text { data. }\end{array}$ & Yes & Medium & $\begin{array}{l}\text { i. Modeling the long-term } \\
\text { behavior profile. } \\
\text { ii. Mostly used for } \\
\text { occupancy modeling. } \\
\text { iii. A stochastic nature } \\
\text { followed by a Markov } \\
\text { property, whereby the future } \\
\text { condition depends only on } \\
\text { the current condition. }\end{array}$ \\
\hline Statistical analysis & Commercial & $\begin{array}{l}\text { Relationship between } \\
\text { the behavior and } \\
\text { other determinants, } \\
\text { or dynamic factors. }\end{array}$ & No & Low & $\begin{array}{l}\text { i. To identify the influential } \\
\text { factors of occupant behavior. } \\
\text { ii. Outputs are being } \\
\text { interconnected by the } \\
\text { occupancy state or the } \\
\text { probability of observed } \\
\text { behavior. }\end{array}$ \\
\hline $\begin{array}{l}\text { Data } \\
\text { mining }\end{array}$ & $\begin{array}{l}\text { Commercial/ } \\
\text { Residential }\end{array}$ & $\begin{array}{l}\text { Categorize the } \\
\text { consistent profile } \\
\text { or/and a systematic } \\
\text { relationship between } \\
\text { the variables. }\end{array}$ & No & Medium & $\begin{array}{l}\text { i. It is the process of } \\
\text { discovering patterns in a } \\
\text { large data set. } \\
\text { ii. To comprehend the } \\
\text { long-term behavior pattern. } \\
\text { iii. Data collection and data } \\
\text { managing are simple to } \\
\text { implement. }\end{array}$ \\
\hline $\begin{array}{l}\text { Agent-based } \\
\text { modeling(ABM) }\end{array}$ & $\begin{array}{l}\text { Commercial/ } \\
\text { Residential }\end{array}$ & $\begin{array}{l}\text { A comprehensive } \\
\text { study of the agent's } \\
\text { relationships, } \\
\text { interactions, and } \\
\text { behavior. }\end{array}$ & Yes & High & $\begin{array}{l}\text { i. Upgrading the simulation } \\
\text { accuracy. } \\
\text { ii. Mostly used in the } \\
\text { simulation-based model } \\
\text { (lack of real data to support } \\
\text { ABM). } \\
\text { iii. It can produce more } \\
\text { precise schedules as input } \\
\text { for BPS (i.e., EnergyPlus). }\end{array}$ \\
\hline
\end{tabular}

From the overall modeling viewpoint, the ABM technique was recommended by the many researchers as the most effective modeling technique [98]. According to [67,68], 
$\mathrm{ABM}$ has the ability to control several behaviors together, and it can represent both grouplevel and individual relations of independent agents. Mostly, the ABM agent is capable of simulating each occupant by unifying characteristics, rules, or data items of the indoor/outdoor environment, as well as enacting a modification in behavior in order to accomplish a specified task. In contrary to other modeling techniques, ABM starts and ends with the agent's perception and purpose. Each agent has individual characteristics that include behaviors and responses. They have the ability to interact with other agents, as well as building surrounding system, which is mainly controlled by user-oriented welldefined rules. These well-defined rules are the groundwork to model agents' behaviors, interactions, and their relationships. However, there is a lack of proper agreement or rules for building a theoretical foundation for ABM model development [12,16]. Still, several problems exist in the latest ABM support behavior studies.

\section{Influential Parameters of Occupant Energy Conservation (EC) Behavior}

Energy conservation (EC) is defined as reducing the consumption of energy by using minimum energy essential services. There are numerous influential parameters that are directly or indirectly related to EC. The key influential parameters of occupant energy conservation (EC) behavior from this review study have been summarized in Figure 9. Usually, the existing approaches for achieving energy conservation goals mainly focus on systems-oriented optimization. Nonetheless, this review focuses on EC from a different perspective by highlighting the building occupant outlook [99], since maintaining the energy consumption by the occupant plays an important role in the building's energy efficiency. For example, inadequate cooling or heating in space will cause occupant dissatisfaction with thermal comfort, and consequently incur increased control over their thermal conditions $[63,70]$. An illustration of such a type of control is the operation of a personal fan or space cooler, which will not only provide better occupant comfort but also consume more energy. The behavior, through occupant control of the built environment, is a usual form of rebound effect (i.e., psychological), which is opposing to building energy conservation in spite of the maximum efficiency accomplished by the building energy systems [100,101]. It also reduces the ability to make accurate predictions of energy requirement in the early design phase. So, it is critical to make design decisions that relate to building energy conservation.

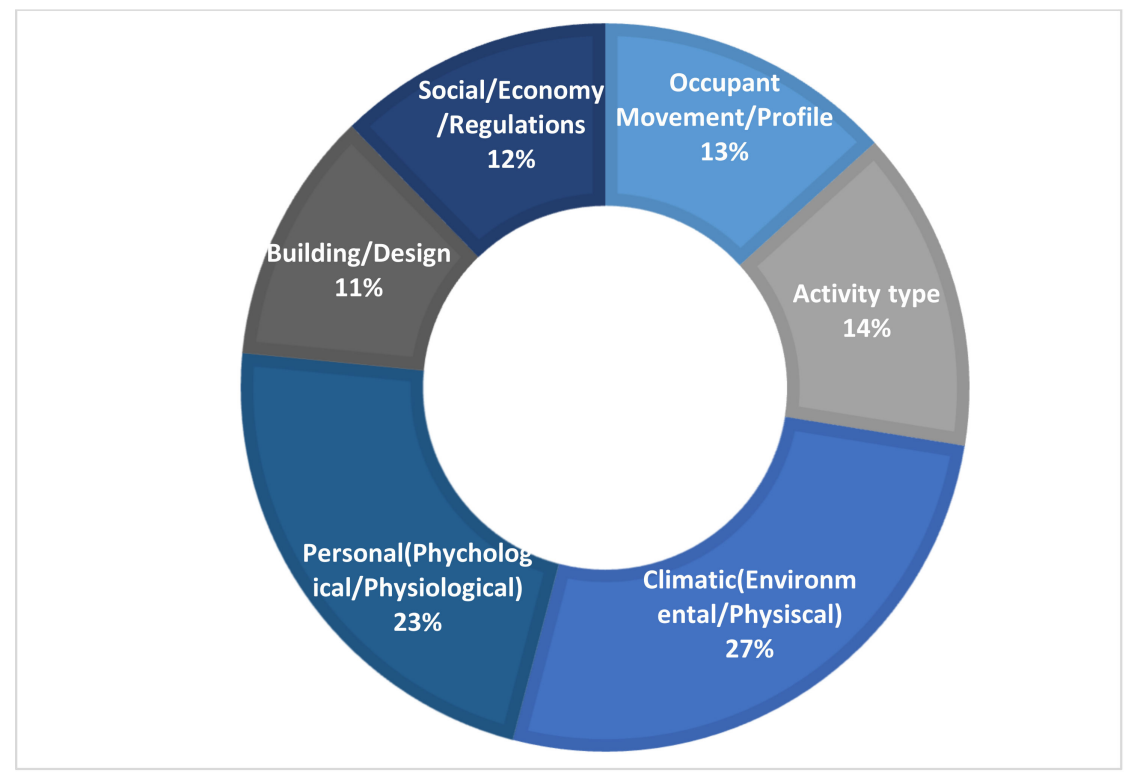

Figure 9. Key influential parameters of occupant energy conservation (EC) behavior. 
In response to the thermal predictability, several studies claim that occupants are more satisfied with a change in thermal conditions in a specific climate [102,103], or occupants feel the necessity to react due to the changing environmental stimulation $[77,89]$. The concept of adaptation is not a new trend; both Persian Plateau courtyard houses and ancient dwellers in Mesa Verde caves migrated indoors in adapting to changing seasonal and diurnal climatic states [68]. This is also the theory behind the human adaptive comfort model, which focuses on the occupants' enhanced tolerance to the indoor environment through thermal adaptation [104,105]. Among building occupants, activity type [56,59] can also be demonstrated as the effective control of their ambient thermal environment with regard to enhancing the the comfort level in the workplace (similar to occupants using personal fans or space heaters) [69]. The behaviors linked with these activities are of primary interest not only to define the indoor climate of occupying individuals' space, but also help define how economic [3,106,107] and socio-personal [105] regulations may affect the style of energy consumption in the building. A limited number of earlier studies indicate the above relationship between the building occupant's behavior and energy performance [108-110], which is also listed in Table 1. On the one hand, an advanced knowledge of occupant behavior will help us to develop a better energy prediction model, which would contribute to better controlled algorithms and systems design [111-113] as well.

\section{Research Gaps for Future Study}

\subsection{Occupancy Centric Space Layout Deployment}

The literature review revealed that most of the behavior research focuses on single prototype buildings, and several city-scale influences have not been studied properly, forming a highly recommended area for future research. Moreover, a few analyses have attempted to assess the impacts of interior space layout on building energy performance [32]. Numerous investigations have shown that layout can significantly affect building energy performance. Besides, the greater parts of these analyses are mixed space designs with different factors; for example, occupant's movement and operation strategy [17], window to wall ratio [114], and shading framework [115]. In addition, at the micro-level, it includes the influence of building interior arrangement in terms of occupant layout preferences, fittings and fixtures, and thermal sensitivities on their energy behavior. It also specified that building space layout may impact occupant's presence and movement, as it might link to the individual action or activities which occur at the specified position within a space [32]. The occupant's presence and movement probability in a specific position is based on several functions (i.e., energy spot distance) of the space that could be simulated. So, occupant-centered design (OCD) techniques, for example, layout preferences, can add to seeing how and why individual occupants consume more energy [32], and this information can guide the plan regarding the interventions to advance energy conservation.

\subsection{Understanding Occupant Behavior in the Context of Developing or Low-Income Economies}

Beyond the fact that several approaches described for the above-mentioned model developments for the building energy monitoring field, still they are related to numerous difficulties and challenges that should be addressed effectively. This review work exposed that most of the existing research focuses on occupancy or occupants from high-income or developed economies, while information on occupants from low-income or developing economies still remains unclear, complex and conflicting. So, it is recommended that people from low-income or developing countries, and their energy-associated behavior in buildings, should be well-understood in terms of economic, social, and other behavioral contexts. Usually, the energy usage of a building is extremely dynamic, and also relies on multiple parameters in terms of socio-economic condition and energy conservation policy. 


\subsection{Lack of Qualitative Behavior Research Compared to Quantitative}

As for a completed analysis, it is inevitable that both quantitative and qualitative information will be utilized. However, most of the current research used quantitative research techniques. In the earlier study, the researcher concentrated more on "what" occupant behavior is instead of "how" and "why" occupant behavior is created [39]. It needs to be noticed that to reduce the effect of human behavior on building energy consumption, it is important to have a comprehensive investigation of the construction pattern of occupant energy behavior, which implies the need for mixed-method techniques. Recently, a few researchers started to understand the significant role of mixed-method techniques when looking into the investigation of the nature of occupant energy behavior [11,39]. It is noted that mixed-method techniques in the field of energy-related occupant behavior are still at their early stages.

\subsection{Exploitation of Survey or Secondary Data and Lack of Real Data Involvement for ABM Validation}

One of the significant approaches within the preceding research studies used national surveys or secondary data and built $\mathrm{ABM}$ approaches without real data involvement [12,70]. A few scholars validated their ABM or behavior models using realistic data [12,69]. Quite often, the model depends on an example or improved model that may prompt questions of whether the simulated agent will play out the behavior in which genuine occupants partake, consequently prompting insufficiency in the model's consistent quality. Just a few model-validation or -verification studies were seen in the earlier works of literature. In [67], a validation study led to the assessment of the ABM via perceptual control theory (PCT). The model's outcomes were seen as practically identical to the field estimations for individual and accumulated projections. However, the model just assumed about thermally adaptive behavior, and the selected behaviors were validated. Putra et al. [69] studied the effect of load shedding on human comfort and behavior. The ABM involved mixed agents/operators and perception capabilities, and a few simulation states. However, just four of the simulation states were analyzed with calculated data, and the test outcomes failed to illustrate an adequate degree of precision.

\subsection{Inclusion of Diverse Category's Buildings and Big Data Stream}

Approximately $85 \%$ of the peer-reviewed studies in this review work focused on the influence of occupant behavior on building energy consumption, particularly in terms of offices and residential buildings (33\% and 52\%, respectively). However, very few articles have examined educational or laboratory buildings. In addition, some other building categories, such as recreational, exhibitions, hotel, clinic, or hospital buildings, have been given sparse attention and require further study [19,31]. On this subject, the big data stream also offers a powerful system to illustrate the full effects of occupant behaviors via a diverse range of data. Besides this, big data will play an important role in automatically generalizing valid, novel, and potentially useful occupancy patterns from a large-scale data set [59]. It might be essential to establish a public process for data reporting to reap the advantages of occupancy data sharing for comprehensive occupancy modeling.

\subsection{BIM Integration with the Existing Occupant Behavior Modeling/Simulation Approach}

Nowadays, BIM implementation for all the advanced stakeholders has been developed because of the plentiful opportunities it offers for their construction schemes, including being value- and time-saving, first-rate and performance-improving, decreasing human resources and clash detection, and allowing greater collaboration and communication. BIM models can be used for behavior engineering analyses while occupant behavior simulation using BIM models is still lacking $[3,45,98,116]$. BIM-incorporated occupant behavior simulation in buildings helps researchers and engineers to identify the design deficiencies, and improve the overall building performance as well as the automation capability [117]. Thus, it is necessary to add another feature to the existing occupant 
behavior study for upgrading the simulation performance. However, occupant behavior studies using BIM technology are relatively lacking, leading to challenges in understanding the consistency between occupants and buildings.

\section{Conclusions}

The building occupants and their behavior are crucial components in our built environment, and their tremendous impact on building energy consumption has recently begun to increase in appreciation. The latest studies on occupant comfort and adaptive control, lighting control, HVAC control, operable window control and shading control are some of the research topics that have started to investigate the occupant behavior or behavioral influences in building energy performance. By reviewing the selected papers published from 2010 to 2019, this study identified the key influential parameters, different behavior modeling approaches, and remaining research gaps by means of systematic analysis. The review analysis revealed that personal (i.e., psychological, physiological), climatic (i.e., environmental, physical), occupant movement, building design, social, and economic criteria are the main features considered by the numerous researchers across the globe. There are mainly five categories of modeling introduced-probabilistic or stochastic, statistical techniques, data mining approach, agent-based modeling (ABM) and others (i.e., BPS, data-driven, ANN, etc.). These are relevant and well-known, and are still frequently used in the latest occupant behavior studies. In addition, as a powerful simulation-based system, recently ABM has become the most popular for the occupant behavior modeling approach in the built environment. The review study also identified six significant research gaps: occupant-centered space layout deployment; occupant behavior needs to be understood in the context of low-income or developing economies; lack of qualitative occupant behavior research compared to quantitative; lack of real data involvement for $\mathrm{ABM}$ validation; behavior studies are required for multi-categories buildings; and BIM integration with an existing occupant behavior modeling/simulation approach.

Technology alone will not accomplish building energy conservation targets. People and their energy-associated behavior in buildings should be evaluated for better energy performance. However, there are a few cases where this understanding of the occupant behavior plays a complete role in the decision-making system. So, the need to furnish occupant behavior modeling has attracted much attention from several researchers. Thus, numerous experimental/modeling studies have been conducted with insightful literature reviews to summarize the key findings. However, due to inadequate data collections, limited or restricted numbers of articles or keywords for review studies and limited scopes, this study provides a systematic review analysis through a keywords search of occupant behavior modeling. This systematic analysis has drawn findings from approximately 83 peer-reviewed articles published between 2010 and 2019 using Scopus and Science Direct databases. Based on the findings, this study has concluded that despite the tremendous achievement in occupant behavior studies for building energy conservation, other significant areas (i.e., the above-mentioned six research gaps) of occupant behavior need to be considered. In addition, comprehensive keyword co-occurrence analysis and research themes were also effective, providing an accurate representation of previous behavior studies while stating the plausible research areas, such as "building energy" and "energy efficiency" due to relatively low publications in these areas.

Despite the contributions of this study, there are limitations that are worth noting regarding the findings. The number of retrieved article records is limited due to the choice of keywords. It is important to note that the keywords used to conduct this study are not exhaustive. Moreover, behavioral factors and other findings of this study are based solely on peer-reviewed journals archived in the Scopus and Science Direct databases. Therefore, this review study has helped to focus the limited purposes of the study. By identifying the major contributions with more wide-ranging determinants of building occupant behavior, these contributions will serve as benchmarks for the relative analysis of the outcome of the study's objective. For example, data collected from the US time-use survey (TUS) would 
be compared to findings from the more comprehensive or realistic contribution of other surveys or data.

Author Contributions: All authors have equally contributed and agreed to the published version of the manuscript.

Funding: The study was fully supported through the funding of full-time UGC-funded Postgraduate Studentship under the auspice of the Department of Building and Real Estate, The Hong Kong Polytechnic University, Hong Kong.

Data Availability Statement: The data presented in this study are available on request from the corresponding authors.

Conflicts of Interest: The authors declare no conflict of interest.

\section{Appendix A. Keywords Frequency, Link and Total Link Strength (2010-2019)}

Table A1. Frequency and total link strength for the selected keywords.

\begin{tabular}{|c|c|c|c|}
\hline Keyword & Frequency & Link & Total Link Strength \\
\hline Energy Utilization & 36 & 47 & 235 \\
\hline Buildings & 32 & 45 & 184 \\
\hline Energy Efficiency & 25 & 45 & 159 \\
\hline Occupant Behavior & 23 & 39 & 143 \\
\hline Office building & 23 & 44 & 138 \\
\hline Behavioral Research & 19 & 38 & 126 \\
\hline Energy Conservation & 17 & 37 & 111 \\
\hline Architectural Design & 17 & 35 & 103 \\
\hline Performance Assessment & 11 & 37 & 87 \\
\hline Simulation & 11 & 35 & 79 \\
\hline Building Performance Simulation & 11 & 33 & 73 \\
\hline Residential Building & 11 & 33 & 71 \\
\hline Stochastic System & 10 & 29 & 59 \\
\hline Survey & 10 & 30 & 56 \\
\hline Computer Simulation & 9 & 28 & 61 \\
\hline Energy Management & 9 & 26 & 55 \\
\hline Air Conditioning & 8 & 32 & 52 \\
\hline Stochastic Model & 8 & 27 & 51 \\
\hline Thermal Comfort & 8 & 28 & 45 \\
\hline Intelligent Building & 7 & 33 & 55 \\
\hline Modeling & 7 & 30 & 52 \\
\hline Indoor Air & 7 & 25 & 37 \\
\hline Building Simulation & 7 & 24 & 41 \\
\hline Regression Analysis & 7 & 24 & 35 \\
\hline Space Heating & 6 & 26 & 40 \\
\hline Building Design & 6 & 17 & 28 \\
\hline Human Behavior & 6 & 21 & 31 \\
\hline Heating & 5 & 26 & 37 \\
\hline Energy Model & 5 & 18 & 34 \\
\hline Energy Plus & 5 & 25 & 39 \\
\hline Data Mining & 5 & 21 & 31 \\
\hline Forecasting & 5 & 24 & 33 \\
\hline Sensitivity Analysis & 5 & 18 & 27 \\
\hline Optimization & 5 & 18 & 25 \\
\hline Window Opening & 5 & 16 & 22 \\
\hline
\end{tabular}

\section{References}

1. Santamouris, M. Minimizing Energy Consumption, Energy Poverty and Global and Local Climate Change in the Built Environment: Innovating to Zero: Causalities and Impacts in a Zero Concept World; Elsevier: Amsterdam, The Netherlands, 2018.

2. Spandagos, C.; $\mathrm{Ng}$, T.L. Equivalent full-load hours for assessing climate change impact on building cooling and heating energy consumption in large Asian cities. Appl. Energy 2017, 189, 352-368. [CrossRef] 
3. Jung, N.; Paiho, S.; Shemeikka, J.; Lahdelma, R.; Airaksinen, M. Energy performance analysis of an office building in three climate zones. Energy Build. 2018, 158, 1023-1035. [CrossRef]

4. Sewell, R.J.; Parry, S.; Millis, S.W.; Wang, N.; Rieser, U.; DeWitt, R. Dating of debris flow fan complexes from Lantau Island, Hong Kong, China: The potential relationship between landslide activity and climate change. (Report). Geomorphology 2015, 248, 205-227. [CrossRef]

5. Evans, M.; Shui, B.A.; Takagi, T. Country Report on Building Energy Codes in Japan (No. PNNL-17849); Pacific Northwest National Lab (PNNL): Richland, WA, USA, 2009.

6. Lopes, L.; Hokoi, S.; Miura, H.; Shuhei, K. Energy efficiency and energy savings in Japanese residential buildings-Research methodology and surveyed results. Energy Build. 2005, 37, 698-706. [CrossRef]

7. Van Dronkelaar, C.; Dowson, M.; Burman, E.; Spataru, C.; Mumovic, D. Corrigendum: A Review of the Energy Performance Gap and Its Underlying Causes in Non-Domestic Buildings. Front. Mech. Eng. 2016, 2, 10. [CrossRef]

8. Chaturvedi, V.; Eom, J.; Clarke, L.; Shukla, P. Long term building energy demand for India: Disaggregating end use energy services in an integrated assessment modeling framework. Energy Policy 2014, 64, 226. [CrossRef]

9. Yarbrough, I.; Sun, Q.; Reeves, D.C.; Hackman, K.; Bennett, R.; Henshel, D.S. Visualizing building energy demand for building peak energy analysis. Energy Build. 2015, 91, 10-15. [CrossRef]

10. Yoshino, H.; Hong, T.; Nord, N. IEA EBC annex 53: Total energy use in buildings-Analysis and evaluation methods. Energy Build. 2017, 152, 124-136. [CrossRef]

11. Balvedi, B.F.; Ghisi, E.; Lamberts, R. A review of occupant behaviour in residential buildings. Energy Build. 2018, 174, 495-505. [CrossRef]

12. Jia, M.; Srinivasan, R.S.; Ries, R.; Weyer, N.; Bharathy, G. A systematic development and validation approach to a novel agent-based modeling of occupant behaviors in commercial buildings. Energy Build. 2019, 199, 352-367. [CrossRef]

13. Hong, T.; D'Oca, S.; Taylor-Lange, S.C.; Turner, W.J.N.; Chen, Y.; Corgnati, S.P. An ontology to represent energy-related occupant behavior in buildings. Part II: Implementation of the DNAS framework using an XML schema. Build. Environ. 2015, 94, 196-205. [CrossRef]

14. Jayathissa, P.; Quintana, M.; Abdelrahman, M.; Miller, C. Humans-as-a-sensor for buildings: Intensive longitudinal indoor comfort models. Buildings 2020, 10, 174. [CrossRef]

15. Eguaras-Martínez, M.; Vidaurre-Arbizu, M.; Martín-Gómez, C. Simulation and evaluation of Building Information Modeling in a real pilot site. Appl. Energy 2014, 114, 475-484. [CrossRef]

16. Zhang, Y.; Bai, X.; Mills, F.P.; Pezzey, J.C.V. Rethinking the role of occupant behavior in building energy performance: A review. Energy Build. 2018, 172, 279-294. [CrossRef]

17. Hong, T.; Taylor-Lange, S.C.; D'oca, S.; Yan, D.; Corgnati, S.P. Advances in research and applications of energy-related occupant behavior in buildings. Energy Build. 2016, 116, 694-702. [CrossRef]

18. Li, J.; Yu, Z.; Haghighat, F.; Zhang, G. Development and improvement of occupant behavior models towards realistic building performance simulation: A review. Sustain. Cities Soc. 2019, 50. [CrossRef]

19. Ioannidis, D. Comparison of detailed occupancy profile generative methods to published standard diversity profiles. Pers. Ubiquitous Comput. 2017, 21, 521-535. [CrossRef]

20. Zhao, J. Design-Build-Operate Energy Information Modeling for Occupant-Oriented Predictive Building Control. Ph.D. Thesis, Carnegie Mellon University, Pittsburgh, PA, USA, 2015.

21. Deblois, J.; Collinge, W.; Bilec, M.; Jones, A.; Schaefer, L. Modeling a Multi-Purpose Public Building with Stochastic Gains and Occupancy Schedules. ASHRAE Trans. 2014, 120, O1-O8.

22. Sun, K.; Hong, T. A simulation approach to estimate energy savings potential of occupant behavior measures. Energy Build. 2017, 136, 43-62. [CrossRef]

23. Debnath, K.B.; Jenkins, D.P.; Patidar, S.; Peacock, A.D. Understanding Residential Occupant Cooling Behaviour through Electricity Consumption in Warm-Humid Climate. Buildings 2020, 10, 78. [CrossRef]

24. Deme Belafi, Z.; Hong, T.; Reith, A. A library of building occupant behaviour models represented in a standardised schema. Energy Effic. 2019, 12, 637-651. [CrossRef]

25. Hong, T.; Sun, H.; Chen, Y.; Taylor-Lange, S.C.; Yan, D. An occupant behavior modeling tool for co-simulation. Energy Build. 2016, 117, 272-281. [CrossRef]

26. Harish, V.S.K.V.; Kumar, A. A review on modeling and simulation of building energy systems. Renew. Sustain. Energy Rev. 2016, 56, 1272-1292. [CrossRef]

27. Hong, T.; Chen, Y.; Belafi, Z.; D'Oca, S. Occupant behavior models: A critical review of implementation and representation approaches in building performance simulation programs. Build. Simul. 2018, 11, 1-14. [CrossRef]

28. Meerbeek, B.W.; de Bakker, C.; de Kort, Y.A.W.; van Loenen, E.J.; Bergman, T. Automated blinds with light feedback to increase occupant satisfaction and energy saving. Build. Environ. 2016, 103, 70-85. [CrossRef]

29. Chen, X.; Wang, Q.; Srebric, J. Occupant feedback based model predictive control for thermal comfort and energy optimization: A chamber experimental evaluation. Appl. Energy 2016, 164, 341-351. [CrossRef]

30. Liang, X.; Hong, T.; Shen, G.Q. Occupancy data analytics and prediction: A case study. Build. Environ. 2016, 102, 179-192. [CrossRef] 
31. Sofia, Y.; Nikos, S.; Emmanuel, K. An Event-Driven Approach for Changing User Behaviour towards an Enhanced Building's Energy Efficiency. Buildings 2020, 10, 183. [CrossRef]

32. Delzendeh, E.; Wu, S.; Lee, A.; Zhou, Y. The impact of occupants' behaviours on building energy analysis: A research review. Renew. Sustain. Energy Rev. 2017, 80, 1061-1071. [CrossRef]

33. Amos Darko, A.P.C.C.; Owusu, E.K.; Antwi-Afari, M.F. Benefits of Green Building: A Literature Review. In Proceedings of the RICS COBRA 2018, RICS HQ, London, UK, 23-24 April 2018. Available online: https:/ / www.rics.org/south-asia/news-insight/ research/conference-papers/benefits-of-green-building-a-literature-review/ (accessed on 19 September 2019).

34. Tober, M. PubMed, ScienceDirect, Scopus or Google Scholar-Which is the best search engine for an effective literature research in laser medicine? Med. Laser Appl. 2011, 26, 139-144. [CrossRef]

35. Zhao, X. A scientometric review of global BIM research: Analysis and visualization. Autom. Constr. 2017, 80, 37. [CrossRef]

36. Su, H.-N.; Lee, P.-C. Mapping knowledge structure by keyword co-occurrence: A first look at journal papers in Technology Foresight. Scientometrics 2010, 85, 65-79. [CrossRef]

37. Prabhakaran, T.; Lathabai, H.H.; Changat, M. Detection of paradigm shifts and emerging fields using scientific network: A case study of Information Technology for Engineering. Technol. Forecast. Soc. Chang. 2015, 91, 124-145. [CrossRef]

38. Santangelo, A.; Yan, D.; Feng, X.; Tondelli, S. Renovation strategies for the Italian public housing stock: Applying building energy simulation and occupant behaviour modelling to support decision-making process. Energy Build. 2018, 167, 269. [CrossRef]

39. Zou, P.; Xu, X.; Sanjayan, J.; Wang, J. A mixed methods design for building occupants' energy behavior research. Energy Build. 2018, 166, 239. [CrossRef]

40. Ren, X.; Yan, D.; Hong, T. Data mining of space heating system performance in affordable housing. Build. Environ. 2015, 89, 1-13. [CrossRef]

41. Jia, M.; Srinivasan, R.S.; Raheem, A.A. From occupancy to occupant behavior: An analytical survey of data acquisition technologies, modeling methodologies and simulation coupling mechanisms for building energy efficiency. Renew. Sustain. Energy Rev. 2017, 68, 525-540. [CrossRef]

42. D'oca, S.; Hong, T.; Langevin, J. The human dimensions of energy use in buildings: A review. Renew. Sustain. Energy Rev. 2018, 81,731-742. [CrossRef]

43. Papadopoulos, S.; Azar, E. Integrating building performance simulation in agent-based modeling using regression surrogate models: A novel human-in-the-loop energy modeling approach. Energy Build. 2016, 128, 214-223. [CrossRef]

44. Tagliabue, L.C.; Manfren, M.; Ciribini, A.L.C.; De Angelis, E. Probabilistic behavioural modeling in building performance simulation-The Brescia eLUX lab. Energy Build. 2016, 128, 119-131. [CrossRef]

45. Hammad, A.W.A. Minimising the Deviation between Predicted and Actual Building Performance via Use of Neural Networks and BIM. Buildings 2019, 9, 131. [CrossRef]

46. Aerts, D.; Minnen, J.; Glorieux, I.; Wouters, I.; Descamps, F. A method for the identification and modelling of realistic domestic occupancy sequences for building energy demand simulations and peer comparison. Build. Environ. 2014, 75, 67-78. [CrossRef]

47. Haldi, F.; Robinson, D. The impact of occupants' behaviour on building energy demand. J. Build. Perform. 2011, 4, 323-338. [CrossRef]

48. Andersen, P.D.; Iversen, A.; Madsen, H.; Rode, C. Dynamic modeling of presence of occupants using inhomogeneous Markov chains. Energy Build. 2014, 69, 213-223. [CrossRef]

49. Barthelmes, V.; Li, R.; Andersen, R.; Bahnfleth, W.; Pcorgnati, S.; Rode, C. Profiling occupant behaviour in Danish dwellings using time use survey data. Energy Build. 2018, 177, 329. [CrossRef]

50. Liisberg, J.; Møller, J.K.; Bloem, H.; Cipriano, J.; Mor, G.; Madsen, H. Hidden Markov Models for indirect classification of occupant behaviour. Sustain. Cities Soc. 2016, 27, 83-98. [CrossRef]

51. Fabi, V.; Andersen, R.K.; Corgnati, S. Verification of stochastic behavioural models of occupants' interactions with windows in residential buildings. Build. Environ. 2015, 94, 371-383. [CrossRef]

52. Guerra Santin, O. Behavioural Patterns and User Profiles related to energy consumption for heating. Energy Build. 2011, 43, 2662-2672. [CrossRef]

53. McLoughlin, F.; Duffy, A.; Conlon, M. Characterising domestic electricity consumption patterns by dwelling and occupant socio-economic variables: An Irish case study. Energy Build. 2011, 48, 240-248. [CrossRef]

54. Fabi, V.; Andersen, R.; Corgnati, S.; Olesen, B. A methodology for modelling energy-related human behaviour: Application to window opening behaviour in residential buildings. Build. Simul. 2013, 6, 415-427. [CrossRef]

55. D'Oca, S.; Fabi, V.; Corgnati, S.; Andersen, R. Effect of thermostat and window opening occupant behavior models on energy use in homes. Build. Simul. 2014, 7, 683-694. [CrossRef]

56. Tabak, V.; de Vries, B. Methods for the prediction of intermediate activities by office occupants. Build. Environ. 2010, 45, 1366-1372. [CrossRef]

57. Yao, J. Modelling and simulating occupant behaviour on air conditioning in residential buildings. Energy Build. 2018, 175, 1-10. [CrossRef]

58. Zhang, X. Smart meter and in-home display for energy savings in residential buildings: A pilot investigation in Shanghai, China. Intell. Build. Int. 2019, 11, 4-26. [CrossRef]

59. D'oca, S.; Hong, T. Occupancy schedules learning process through a data mining framework. Energy Build. 2015, 88, 395-408. [CrossRef] 
60. Ashouri, M.; Haghighat, F.; Fung, B.C.M.; Lazrak, A.; Yoshino, H. Development of building energy saving advisory: A data mining approach. Energy Build. 2018, 172, 139-151. [CrossRef]

61. Zhao, J.; Lasternas, B.; Lam, K.P.; Yun, R.; Loftness, V. Occupant behavior and schedule modeling for building energy simulation through office appliance power consumption data mining. Energy Build. 2014, 82, 341-355. [CrossRef]

62. Zhou, H.; Qiao, L.; Jiang, Y.; Sun, H.; Chen, Q. Recognition of air-conditioner operation from indoor air temperature and relative humidity by a data mining approach. Energy Build. 2016, 111, 233-241. [CrossRef]

63. Wang, J.; Zhu, J.; Ding, Z.; Zou, P.X.W.; Li, J. Typical energy-related behaviors and gender difference for cooling energy consumption. J. Clean. Prod. 2019, 238. [CrossRef]

64. Yu, Z.; Fung, B.C.M.; Haghighat, F.; Yoshino, H.; Morofsky, E. A systematic procedure to study the influence of occupant behavior on building energy consumption. Energy Build. 2011, 43, 1409-1417. [CrossRef]

65. Azar, E.; Menassa, C.C. Agent-Based Modeling of Occupants and Their Impact on Energy Use in Commercial Buildings. J. Comput. Civ. Eng. 2012, 26, 506-518. [CrossRef]

66. Kashif, A.; Ploix, S.; Dugdale, J.; Le, X.H.B. Simulating the dynamics of occupant behaviour for power management in residential buildings. Energy Buildings 2013, 56, 85-93. [CrossRef]

67. Langevin, J.; Wen, J.; Gurian, P.L. Simulating the human-building interaction: Development and validation of an agent-based model of office occupant behaviors. Build. Environ. 2015, 88, 27-45. [CrossRef]

68. Lee, Y.S.; Malkawi, A.M. Simulating multiple occupant behaviors in buildings: An agent-based modeling approach. Energy Build. 2014, 69, 407. [CrossRef]

69. Putra, H.; Andrews, C.; Senick, J. An agent-based model of building occupant behavior during load shedding. Build. Simul. 2017, 10, 845-859. [CrossRef]

70. Azar, E.; Nikolopoulou, C.; Papadopoulos, S. Integrating and optimizing metrics of sustainable building performance using human-focused agent-based modeling. Appl. Energy 2016, 183, 926-937. [CrossRef]

71. Ding, Z.; Hu, T.; Li, M.; Xu, X.; Zou, P.X.W. Agent-based model for simulating building energy management in student residences. Energy Build. 2019, 198, 11-27. [CrossRef]

72. Langevin, J.; Wen, J.; Gurian, P.L. Quantifying the human-building interaction: Considering the active, adaptive occupant in building performance simulation. Energy Build. 2016, 117, 372-386. [CrossRef]

73. Azar, E.; Menassa, C.C. Evaluating the impact of extreme energy use behavior on occupancy interventions in commercial buildings. Energy Build. 2015, 97, 205-218. [CrossRef]

74. Chen, J.; Taylor, J.E.; Wei, H.-H. Modeling building occupant network energy consumption decision-making: The interplay between network structure and conservation. Energy Build. 2012, 47, 515-524. [CrossRef]

75. Azar, E.; Al Ansari, H. Multilayer Agent-Based Modeling and Social Network Framework to Evaluate Energy Feedback Methods for Groups of Buildings. J. Comput. Civ. Eng. 2017, 31. [CrossRef]

76. Pisello, A.L.; Asdrubali, F. Human-based energy retrofits in residential buildings: A cost-effective alternative to traditional physical strategies. Appl. Energy 2014, 133, 224-235. [CrossRef]

77. Peng, Y.; Nagy, Z.; Schlüter, A. Temperature-preference learning with neural networks for occupant-centric building indoor climate controls. Build. Environ. 2019, 154, 296-308. [CrossRef]

78. Abuimara, T.; O’Brien, W.; Gunay, B.; Carrizo, J.S. Towards occupant-centric simulation-aided building design: A case study. Build. Res. Inf. 2019, 47, 866-882. [CrossRef]

79. Lopes, M.A.R.; Antunes, C.H.; Reis, A.; Martins, N. Estimating energy savings from behaviours using building performance simulations. Build. Res. Inf. 2017, 45, 303-319. [CrossRef]

80. Sharmin, T.; Gül, M.; Al-Hussein, M. A user-centric space heating energy management framework for multi-family residential facilities based on occupant pattern prediction modeling. Build. Simul. 2017, 10, 899-916. [CrossRef]

81. Zhu, P.; Gilbride, M.; Yan, D.; Sun, H.; Meek, C. Lighting energy consumption in ultra-low energy buildings: Using a simulation and measurement methodology to model occupant behavior and lighting controls. Build. Simul. 2017, 10, 799-810. [CrossRef]

82. Belafi, Z.; Hong, T.; Reith, A. Smart building management vs. intuitive human control—Lessons learnt from an office building in Hungary. Build. Simul. 2017, 10, 811-828. [CrossRef]

83. Feng, X.; Yan, D.; Yu, R.; Gao, Y. Investigation and modelling of the centralized solar domestic hot water system in residential buildings. Build. Simul. 2017, 10, 87-96. [CrossRef]

84. Leroy, Y.; Yannou, B. An activity-based modelling framework for quantifying occupants' energy consumption in residential buildings. Comput. Ind. 2018, 103, 1-13. [CrossRef]

85. Hu, S.; Yan, D.; Qian, M. Using bottom-up model to analyze cooling energy consumption in China's urban residential building. Energy Build. 2019, 202. [CrossRef]

86. Yu, C.; Du, J.; Pan, W. Improving accuracy in building energy simulation via evaluating occupant behaviors: A case study in Hong Kong. Energy Build. 2019, 202. [CrossRef]

87. Zahiri, S.; Elsharkawy, H. Towards energy-efficient retrofit of council housing in London: Assessing the impact of occupancy and energy-use patterns on building performance. Energy Build. 2018, 174, 672-681. [CrossRef]

88. Ben, H.; Steemers, K. Energy retrofit and occupant behaviour in protected housing: A case study of the Brunswick Centre in London. Energy Build. 2014, 80, 120-130. [CrossRef] 
89. Lim, J. Cooling Energy Implications of Occupant Factor in Buildings under Climate Change. Sustainability 2017, 9, 2039. [CrossRef]

90. Wang, W.; Chen, J.; Song, X. Modeling and predicting occupancy profile in office space with a Wi-Fi probe-based Dynamic Markov Time-Window Inference approach. Build. Environ. 2017, 124, 130-142. [CrossRef]

91. D'Oca, S.; Gunay, H.B.; Gilani, S.; O'Brien, W. Critical review and illustrative examples of office occupant modelling formalisms. Build. Serv. Eng. Res. Technol. 2019, 40, 732-757. [CrossRef]

92. Yan, D. Occupant behavior modeling for building performance simulation: Current state and future challenges. Energy Build. 2015, 107, 264-278. [CrossRef]

93. Gunay, H.B.; O’Brien, W.; Beausoleil-Morrison, I. A critical review of observation studies, modeling, and simulation of adaptive occupant behaviors in offices. Build. Environ. 2013, 70, 31. [CrossRef]

94. Diao, L.; Sun, Y.; Chen, Z.; Chen, J. Modeling energy consumption in residential buildings: A bottom-up analysis based on occupant behavior pattern clustering and stochastic simulation. Energy Build. 2017, 147, 47-66. [CrossRef]

95. Haldi, F.; Robinson, D. On the behaviour and adaptation of office occupants. Build. Environ. 2008, 43, 2163-2177. [CrossRef]

96. Hong, T.; Yan, D.; D'Oca, S.; Chen, C.-F. Ten questions concerning occupant behavior in buildings: The big picture. Build. Environ. 2017, 114, 518-530. [CrossRef]

97. Labeodan, T.; Aduda, K.; Boxem, G.; Zeiler, W. On the application of multi-agent systems in buildings for improved building operations, performance and smart grid interaction-A survey. Renew. Sustain. Energy Rev. 2015, 50, 1405-1414. [CrossRef]

98. Micolier, A.; Taillandier, F.; Taillandier, P.; Bos, F. Li-BIM, an agent-based approach to simulate occupant-building interaction from the Building-Information Modelling. Eng. Appl. Artif. Intell. 2019, 82, 44-59. [CrossRef]

99. Sipowicz, E.; Sulaiman, H.; Filippín, C.; Pipa, D. Dwelling's energy saving through the experimental study and modeling of technological interventions in a cold temperate climate of Argentina. Energy Effic. 2018, 11, 975-995. [CrossRef]

100. Wei, S.; Jones, R.; de Wilde, P. Driving factors for occupant-controlled space heating in residential buildings. Energy Buildings 2014, 70, 36-44. [CrossRef]

101. Fotopoulou, E. Providing Personalized Energy Management and Awareness Services for Energy Efficiency in Smart Buildings. Sensors 2017, 17. [CrossRef]

102. Castaldo, V.L.; Pigliautile, I.; Rosso, F.; Cotana, F.; De Giorgio, F.; Pisello, A.L. How subjective and non-physical parameters affect occupants' environmental comfort perception. Energy Build. 2018, 178, 107-129. [CrossRef]

103. Hu, S.; Yan, D.; Cui, Y.; Guo, S. Urban residential heating in hot summer and cold winter zones of China-Status, modeling, and scenarios to 2030. Energy Policy 2016, 92, 158-170. [CrossRef]

104. Deuble, M.P.; de Dear, R.J. Mixed-mode buildings: A double standard in occupants' comfort expectations. Build. Environ. 2012, 54, 53-60. [CrossRef]

105. Von Grabe, J. A preliminary cognitive model for the prediction of energy-relevant human interaction with buildings. Cogn. Syst. Res. 2018, 49, 65-82. [CrossRef]

106. Wierzba, A.; Morgenstern, M.; Meyer, S.; Ruggles, T.; Himmelreich, J. A study to optimize the potential impact of residential building energy audits. Energy Effic. 2011, 4, 587-597. [CrossRef]

107. Bellia, L.; Borrelli, M.; De Masi, R.F.; Ruggiero, S.; Vanoli, G.P. University building: Energy diagnosis and refurbishment design with cost-optimal approach. Discussion about the effect of numerical modelling assumptions. J. Build. Eng. 2018, 18, 1-18. [CrossRef]

108. Ferroukhi, M.; Djedjig, R.; Limam, K.; Belarbi, R. Hygrothermal behavior modeling of the hygroscopic envelopes of buildings: A dynamic co-simulation approach. Build. Simul. 2016, 9, 501-512. [CrossRef]

109. Ferrantelli, A.; Ahmed, K.; Pylsy, P.; Kurnitski, J. Analytical modelling and prediction formulas for domestic hot water consumption in residential Finnish apartments. Energy Build. 2017, 143, 53-60. [CrossRef]

110. Popoola, O.; Munda, J.; Mpanda, A.; Popoola, A.P.I. Comparative analysis and assessment of ANFIS-based domestic lighting profile modelling. Energy Build. 2015, 107, 294-306. [CrossRef]

111. Abdallah, F.; Basurra, S.; Gaber, M.M. A Non-Intrusive Heuristic for Energy Messaging Intervention Modeled Using a Novel Agent-Based Approach. IEEE Access 2019, 7, 1627-1646. [CrossRef]

112. Von Grabe, J. Potential of artificial neural networks to predict thermal sensation votes. Appl. Energy 2016, 161, 412-424. [CrossRef]

113. Chen, J.; Jain, R.K.; Taylor, J.E. Block Configuration Modeling: A novel simulation model to emulate building occupant peer networks and their impact on building energy consumption. Appl. Energy 2013, 105, 358-368. [CrossRef]

114. Troup, L.; Phillips, R.; Eckelman, M.; Fannon, D. Effect of window-to-wall ratio on measured energy consumption in US office buildings. Energy Build. 2019, 203. [CrossRef]

115. Han, Y.; Taylor, J.E.; Pisello, A.L. Exploring mutual shading and mutual reflection inter-building effects on building energy performance. Appl. Energy 2017, 185, 1556-1564. [CrossRef]

116. Cheng, J.C.P.; Gan, V.J. Integrating Agent-Based Human Behavior Simulation with Building Information Modeling for Building Design. IJET 2013, 5, 473-477. [CrossRef]

117. Eguaras-Martínez, M.; Martín-Gómez, C.; Vidaurre-Arbizu, M.; Brennan, T.; Krinidis, S.; Ioannidis, D.; Tzovaras, D. Architectural simulation of the integration of Building Information Modelling (BIM) \& Business Process Modelling (BPM). In Proceedings of the eWork and eBusiness in Architecture, Engineering and Construction; CRC Press: Boca Raton, FL, USA, 2014. 